\title{
Interactions Between Fungal Hyaluronic Acid and Host CD44 Promote Internalization by Recruiting Host Autophagy Proteins to Forming Phagosomes
}

\section{Graphical Abstract}

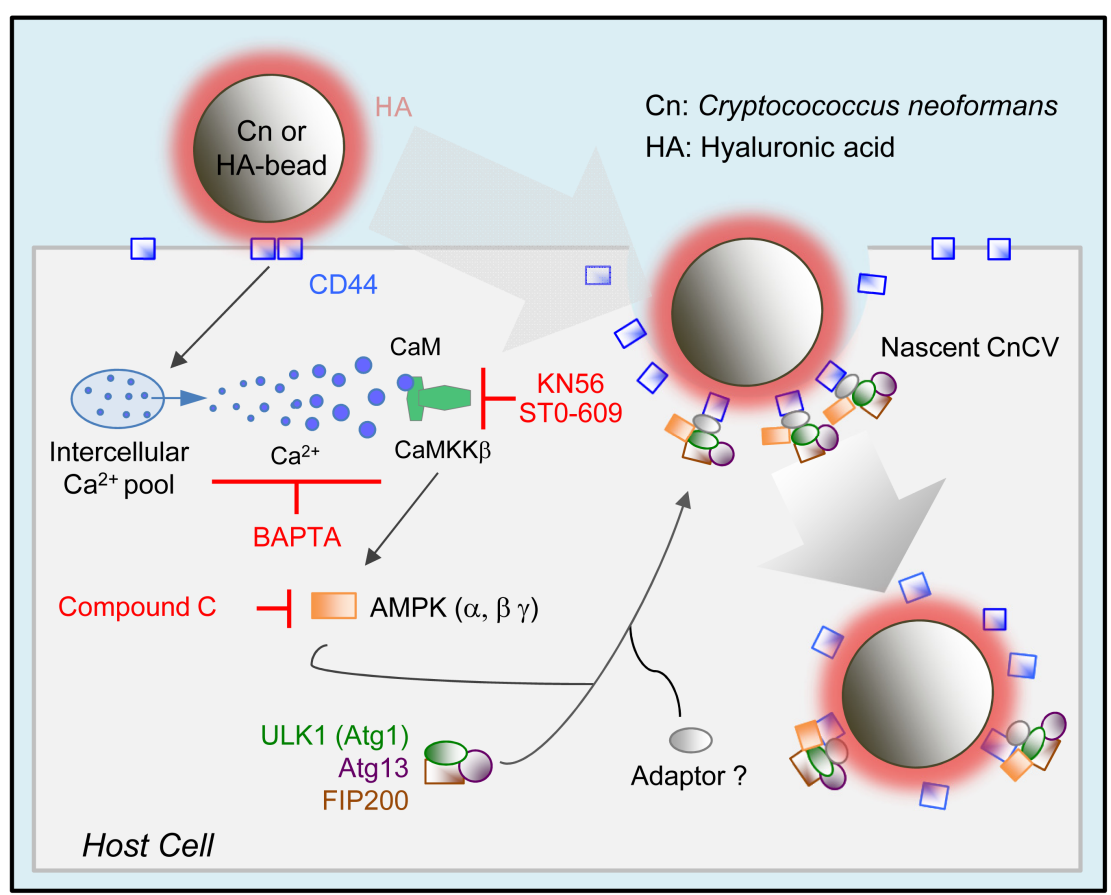

Highlights

- Fungal HA interactions with host cells drive a novel non-canonical, ligand-induced, autophagy pathway in phagocytic cells

- Cryptococcus neoformans recruits host CD44, together with AIC components and regulatory proteins, to forming phagocytic cups to initiate host cell internalization

- Fungal HA interactions with CD44 on host cell surfaces elevate intracellular $\mathrm{Ca}^{2+}$ concentrations, leading to activation of CaMKK $\beta$

- $\mathrm{A} \mathrm{Ca}^{2+}$-CaMKK $\beta-A M P K-U L K 1$ signaling axis is involved in $\mathrm{HA}$ and $\mathrm{CD} 44$ induced autophagy protein recruitment during $\mathrm{C} n$ internalization

\section{Authors}

Sheng Li Ding, Aseem Pandey, Xuehuan Feng, ... , Qing-Ming Qin, Thomas A. Ficht, Paul de Figueiredo

\section{Correspondence}

qmqin@jlu.edu.cn (Q.M.Q.), tficht@tamu.edu (T.A.F.), pjdefigueiredo@tamu.edu (P.d.F.) In Brief

Ding et al. reveal that interactions between fungal hyaluronic acid (HA) and host $\mathrm{CD} 44$ activate a $\mathrm{Ca}^{2+}$ CaMKK $\beta$-AMPK-ULK1 signaling pathway that recruits autophagy initiation complex components to forming phagosomes to drive fungal internalization. 


\section{Interactions between fungal hyaluronic acid and host CD44 promote} 2 internalization by recruiting host autophagy proteins to forming phagosomes

4 Sheng Li Ding ${ }^{1,2,6}$, Aseem Pandey ${ }^{2,3,6}$, Xuehuan Feng ${ }^{2,6}$, Jing Yang ${ }^{2}$, Luciana Fachini da Costa ${ }^{2,4}$, 5 Roula Mouneimne ${ }^{3}$, Allison Rice-Ficht ${ }^{4}$, Samantha L.Bell ${ }^{5}$, Robert O. Watson ${ }^{5}$, Kristin Patrick ${ }^{5}$, Qing-Ming Qin $^{1,2 *}$, Thomas A. Ficht ${ }^{3^{*}}$, Paul de Figueiredo ${ }^{2,3,7 *}$

${ }^{1}$ College of Plant Sciences \& Key Laboratory of Zoonosis Research, Ministry of Education, Jilin

9 University, Changchun 130062, Jilin, China; Department of Plant Pathology, College of Plant

10 Protection, Henan Agricultural University, Zhengzhou 450002, Henan, China

$11{ }^{2}$ Department of Microbial Pathogenesis and Immunology, Texas A\&M Health Science Center,

12 Norman Borlaug Center, Texas A\&M University, College Station, Texas 77843, USA

$13{ }^{3}$ Department of Veterinary Pathobiology, Texas A\&M University, College Station, Texas 77843,

14 USA

$15{ }^{4}$ Department of Molecular and Cellular Medicine, College of Medicine, Texas A\&M Health

16 Science Center, College Station, Texas 77843, USA

$17{ }^{5}$ Department of Microbial Pathogenesis and Immunology, Texas A\&M Health Science Center,

18 Bryan, Texas 77807, USA

$19{ }^{6}$ These authors contributed equally

$20 \quad{ }^{7}$ Lead contact

21 *Correspondence: qmqin@jlu.edu.cn (Q.M.Q.), tficht@tamu.edu (T.A.F.), pjdefigueiredo@tamu.edu (P.d.F.) 


\section{Summary}

3 Phagocytosis and autophagy play critical roles in immune defense. Cryptococcus neoformans $(\mathrm{Cn})$,

4 a fungal pathogen that causes fatal infection, subverts the host autophagy initiation complex (AIC)

5 and its upstream regulatory proteins, to promote its phagocytosis and intracellular parasitism of

6 host cells. The mechanisms by which the pathogen engages host AIC proteins remain obscure.

7 Here, we show that the recruitment of host AIC proteins to forming phagosomes is dependent

8 upon the activity of CD44, a host cell surface receptor that engages fungal hyaluronic acid (HA).

9 This interaction elevates intracellular $\mathrm{Ca}^{2+}$ concentrations and activates $\mathrm{CaMKK} \beta$ and its

10 downstream target AMPK $\alpha$, which results in activation of ULK1 and the recruitment of AIC

11 components. Moreover, we demonstrate that HA-coated beads efficiently recruit AIC components

12 to phagosomes. Taken together, these findings show that fungal HA plays a critical role in

13 directing the internalization and productive intracellular membrane trafficking of a fungal

14 pathogen of global importance.

15 


\section{Introduction}

3 Autophagy is an orderly "self-eating" process in cells that coordinates the degradation of cellular components. Various types of autophagy have been described, including macrophagy, microphagy, mitophagy, chaperone-mediated autophagy and xenophagy (Galluzzi et al., 2017; Khandia et al., 2019; Kirkin and Rogov, 2019). Some pathogens subvert autophagic machinery to promote their intracellular survival and replication (Case et al., 2016; de Figueiredo and Dickman, 2016; de Figueiredo et al., 2015; Dickman et al., 2017; Pandey et al., 2018). Several signaling pathways control the onset, duration and outcome of autophagy induction in mammalian cells (Abada and Elazar, 2014; Rubinsztein et al., 2012). Pathways that include components of the autophagy

11 initiation complex (AIC), including AMPK, ULK1, ATG13, FIP200 and ATG9, play important

12 roles in these processes (Ganley et al., 2009; Hosokawa et al., 2009; Jung et al., 2009). For example, AMPK or ULK1 signaling can regulate ATG9 recruitment to nascent phagosome or

14 autophagosome membranes (Mack et al., 2012). This recruitment process is believed to contribute

15 to the elongation of the autophagosomal membrane (Mack et al., 2012).

Cryptococcus neformans $(\mathrm{Cn})$ is a pathogen of global consequence that causes fatal fungal meningoencephalitis worldwide (Kozubowski and Heitman, 2012; Olszewski et al., 2010; Sabiiti and May, 2012). Cn is particularly pernicious in immunocompromised individuals, where lethal infection constitutes a significant risk (Warkentien and Crum-Cianflone, 2010). Cn can survive, replicate and persist in both intracellular and extracellular environments within mammalian hosts

22 (Garcia-Rodas and Zaragoza, 2012). However, the molecular mechanisms that control intracellular parasitism remain poorly understood (Evans et al., 2018; Zaragoza, 2019). Towards addressing this issue, we reported a functional analysis of host factors that regulate the infection, intracellular replication, and non-lytic release of $\mathrm{Cn}$ from host cells (Qin et al., 2011). We extended these findings by performing a phosphoproteomic analysis of the host response to $\mathrm{Cn}$ infection (Pandey et al., 2017). This analysis demonstrated that host AIC proteins, and upstream regulatory molecules, contribute to the internalization and intracellular replication of the pathogen in macrophages (Pandey et al., 2017). This work also raised questions about the cellular and molecular mechanisms by which these proteins contribute to this phenotype. 
1 The internalization of $\mathrm{Cn}$ into host cells is regulated, in part, by interactions between fungal

2 components and host associated CD44, a major receptor for hyaluronic acid (HA) in mammalian

3 cells (Jong et al., 2012; Jong et al., 2008). Moreover, CD44 has been shown to control

4 phagocytosis of the pathogen (Jong et al., 2012; Jong et al., 2008). Interestingly, mice deficient for

5 CD44 display reduced susceptibility to infection (Jong et al., 2012). The deficiency in

6 phagocytosis accounts for this phenotype, leaving open the question of the mechanism by which

7 CD44 controls fungal internalization. Here, we show that $\mathrm{Cn}$ phagocytosis by macrophages occurs

8 by a novel mechanism whereby AIC proteins, including ULK1, ATG9 and ATG13, as well as the

9 key upstream signaling component $\mathrm{AMPK} \alpha$, are recruited to forming phagosomes to promote the

10 phagocytosis of the pathogen in a CD44-dependent fashion. Interaction of fungal HA and host

$11 \mathrm{CD} 44$ activates a $\mathrm{Ca}^{2+}-\mathrm{CaMKK} \beta$ (calcium/calmodulin-dependent protein kinase kinase $\beta$ subunit)-

12 AMPK-ULK1 signaling axis that supports Cn internalization into host cells. Taken together, our

13 findings uncover unexpected roles for HA-CD44 interactions in conferring susceptibility to fungal

14 infection and open up new avenues for therapeutic intervention for a fungal pathogen of global

15 importance.

\section{Results}

\section{Cn recruits host $\mathrm{AIC}$ components to forming Cn-containing phagosomes}

20 To test the hypothesis that $\mathrm{Cn}$ infection of macrophages promotes the formation of a physical 21 complex that contains AIC components, we used Forster Resonance Energy Transfer (FRET)

22 imaging microscopy, which detects close molecular associations ( $<10 \mathrm{~nm}$ ) (Irving et al., 2014), to

23 measure such interactions. The quenching of fluorescence in the donor fluorophore of a FRET pair

24 accompanies the establishment of a close physical association between the pairs (Irving et al., 25 2014). We measured photon transfer between antibody labeled ATG13 and ULK1 or AMPK $\alpha$ and

26 FIP200 in infected and uninfected RAW264.7 macrophages. We observed significant increases in 27 the amount of FRET between these proteins in infected cells (Figure S1A-D). However, 28 comparable FRET interactions were not observed in controls that were stained with a single label, 29 or in uninfected samples (Figure S1A-D), thereby indicating the establishment of a close 30 association between these proteins during infection. 
1 Recruitment of AIC components to forming phagosomes containing Cn is galectin 8 2 independent

3 Previous studies have shown that galectin 8 , a $\beta$-galactoside-binding lectin, monitors endosomal 4 and lysosomal integrity by binding to host glycans on damaged pathogen-containing vacuoles.

5 This binding drives the ubiquitin-dependent recruitment of autophagy adaptor proteins (e.g., 6 NDP52) and microtubule associated light chain kinase 3 (LC3), which in turn, promotes the 7 recruitment of autophagosome biogenesis proteins to damaged pathogen-containing vacuoles 8 (Thurston et al., 2012). Galectin 8 mediated autophagosomal targeting is relevant to the observed 9 recruitment of AIC components to phagocytic cups because $\mathrm{Cn}$ containing vacuoles (CnCVs) are 10 permeabilized after phagocytosis by macrophages (Johnston and May, 2010). Moreover, nascent 11 CnCVs in infected macrophages recruit host LC3 (Fig. S1E) (Nicola et al., 2012; Qin et al., 2011), 12 thereby implicating trafficking pathways that recruit LC3 to phagosomal membranes in controlling 13 the intracellular lifestyle of $\mathrm{Cn}$. With these ideas in mind, we tested the hypothesis that AIC 14 recruitment to nascent $\mathrm{CnCVs}$ is associated with galectin 8 recruitment to these subcellular 15 structures. We infected RAW264.7 macrophages that express a GFP-tagged variant of galectin 8 16 (GFP-Gal8) with $\mathrm{Cn}$, and then used immunofluorescence microscopy (IFM) to determine whether 17 GFP-Gal8 colocalized with AMPK $\alpha$ on nascent phagosomes containing Cn cells. We found that 18 GFP-Gal8 did not display quantitative colocalization with either AMPK $\alpha$ or nascent phagosomes 19 (Figure S1F). Consistent with these observations was the finding that UBEI-41, a potent and cell20 permeable inhibitor of ubiquitin E1 activity (Yang et al., 2007), when added to macrophages at 21 non-toxic doses $(30 \mu \mathrm{M})$, did not diminish AIC recruitment to CnCVs (Figure S1G). Importantly, 22 the inhibitor was washed out of the host cell culture media before $\mathrm{Cn}$ infection, thereby ensuring 23 that the drug only targeted host cell components in these experiments. In addition, we found that 24 nascent phagosomes decorated with the AIC component ULK1 colocalized with the endosomal 25 marker EEA1 (Figure S1H). We also found that calreticulin, a $\mathrm{Ca}^{2+}$ binding ER (endoplasmic 26 reticulum) protein, was recruited to phagocytic cups and nascent CnCVs (Figure S1I), and that 27 LC3 displayed minimal colocalization with AIC components on nascent CnCVs (Figure S1J, left). $28 \mathrm{CnCVs}$ in $\mathrm{B} 6 \mathrm{~J} 2$ macrophages expressing dominant negative variants of the AIC regulatory 29 component AMPK $\alpha$ recruited less LC3 (Figure S1J, right) and AIC components (Figure S1K), 30 thereby suggesting that AIC recruitment to nascent Cn-containing phagosomes and LC3 31 recruitment to phagosome membranes could be morphologically and genetically dissected. Taken 
1 together, these findings indicated that recruitment of AIC to the nascent phagosomes and the

2 induction of autophagy were galectin 8-independent events.

4 A non-proteinaceous and/or non-capsular component controls AIC recruitment to nascent 5 CnCVs

6 The observation that AIC and AIC regulatory components were recruited to forming phagosomes

7 during $\mathrm{Cn}$ infection of macrophages encouraged us to determine the mechanism of recruitment.

8 We infected host cells with live or dead $\mathrm{Cn}$ and found that fungal viability was not required for

9 AIC recruitment because nascent $\mathrm{CnCVs}$ containing the heat-killed (HK) organism also efficiently

10 recruited these proteins (Figure 1A). These observations were consistent with the hypothesis that

11 non-proteinaceous, Cn-associated molecules activate the host AIC pathway.

$13 \mathrm{Cn}$ is encased in a carbohydrate-enriched capsule that is essential for intracellular parasitism and 14 virulence (O'Meara and Alspaugh, 2012). To test the hypothesis that capsular components direct 15 the recruitment of AIC and AIC regulatory proteins to forming phagosomes, we infected host cells 16 with an acapsular mutant of Cn (Cap59), which displays defects in extracellular trafficking of 17 glucuronoxylomannan (GXM) (Garcia-Rivera et al., 2004), and analyzed AIC recruitment to 18 phagosomes containing cap59 strains. We found that forming and formed phagosomes that 19 contained the acapsular strain also efficiently recruited AIC proteins (Figure 1B-D, Figure S2A-

20 C). AIC components showed close associations with internalized $\mathrm{Cn}$ cells as detected by indirect 21 immunofluorescence with anti-capsular monoclonal antibody 18B7 (Garcia-Rivera et al., 2004)

22 (Figure S2D). Moreover, infection of host cells with live wild-type (WT) Cn resulted in the 23 activation by phosphorylation of host cell ULK1 (Ser555), a key component of the AIC, and 24 activation of the AIC regulatory protein AMPK $\alpha$ (Thr172) (Pandey et al., 2017). Similarly, the 25 acapsular mutant and HK Cn also activated host AMPK $\alpha$ (Thr172) and/or ULK1 (Figure 1E-H, 26 Figure S2E). However, when $S$. cerevisiae was incubated with host cells, similar AIC recruitment 27 was not observed (Figure S2F), suggesting that phagocytosis of yeast cells by macrophages 28 involves the participation of a distinct mechanism. Taken together, these findings suggested that 29 Cn-specific, non-proteinaceous, non-capsular components activated the host AMPK-ULK1 30 signaling axis and promoted AIC recruitment to forming phagosomes. 


\section{CD44-deficient host cells fail to recruit AIC proteins to forming phagosomes}

2 CD44 regulates fungal internalization (Jong et al., 2012; Jong et al., 2008). This observation raised

3 the intriguing possibility that CD44 interactions with other host cell components may also control

4 the recruitment of AIC components to nascent CnCVs. To test this hypothesis, we first used

5 fluorescence microscopy to determine whether CD44 colocalized with Cn cells or was recruited to

6 forming or formed nascent CnCVs. We also tested whether CD44 deficient macrophages recruited

7 AIC components to the nascent pathogen-containing phagosomes. We found that forming or

8 formed phagosomes containing both capsular or acapsular strains displayed strong colocalization

9 with CD44 (Figure 2A), and that CD44 was enriched at sites of contact between the pathogen and

10 the host cell surface during a time course of infection (Figure S2G). Next, we tested whether

11 CD44 colocalized with AIC regulatory AMPK and AIC components on nascent CnCVs. We

12 found that AIC components, including ATG9 and ULK1, colocalized with CD44 on nascent

13 CnCVs (Figure 2B-C). Compared to $\mathrm{CD}_{4} 4^{+/+}$bone-marrow derived macrophage (BMDM)

14 controls, AMPK $\alpha$ showed reduced colocalization in CD44 ${ }^{-/-}$BMDMs (Figure 2D). As a result, Cn

15 internalization in CD44-deficient cells was reduced (Figure 2E-F), indicating that host cell CD44

16 is required for $\mathrm{Cn}$ internalization, and that activation of the AMPK-ULK1 signaling axis and AIC

17 recruitment is CD44-dependent.

19 cps1 deletion mutants fail to recruit AIC components to nascent CnCVs

20 HA, a component of the $\mathrm{Cn}$ cell wall, is known to interact with CD44, an HA receptor on host

21 cells, and to promote the internalization of the pathogen into human and murine brain 22 microvascular endothelial cells (BMECs) (Jong et al., 2012; Jong et al., 2008). This observation

23 raised the intriguing possibility that HA-CD44 interactions may promote the recruitment of AIC

24 components to nascent CnCVs. To test this hypothesis, we first examined the internalization of $\mathrm{Cn}$

25 strains that harbor mutations in cps 1, a gene that encodes hyaluronic synthase (Jong et al., 2007).

26 Consistent with previous findings where $\mathrm{Cn}$ displayed reduced association with CD44-depleted

27 murine BMECs compared to controls (Jong et al., 2012), we found that deletion of cps 1 displayed

28 reduced internalization of $\mathrm{Cn}$ into host macrophages (Figure 3A-C, Figure S3A). Next, we used

29 fluorescence microscopy to determine whether cpsl-deficient $\mathrm{Cn}$ strains recruited AIC

30 components to nascent pathogen-containing phagosomes. We found the mutant strain displayed

31 reduced recruitment compared to WT controls (Figure 3D). However, nascent CnCVs that 
1 contained strain C558 (cps1 1 ::CPS1), in which the cps1 mutation was complemented with a WT copy of the gene (Jong et al., 2008), displayed higher levels of AIC recruitment than their CPS1deficient counterparts (Figure 3D). These data suggested that HA, the product of cps 1 activity, contributed to directing the recruitment of AIC components to nascent $\mathrm{CnCVs}$, and implicated a role for $\mathrm{CD} 44$, the dominant $\mathrm{HA}$ receptor on macrophages, in regulating this process.

\section{Pathogen-derived HA drives interactions between CD44 and AIC components}

To test whether HA was sufficient to induce CD44-mediated recruitment of AIC components to nascent $\mathrm{CnCVs}$, we determined whether HA-coated beads induced similar recruitment to forming phagosomes. For these experiments, we covalently coupled HA to polystyrene beads and then incubated the HA-coupled beads with RAW264.7 macrophages for various lengths of time. We also used antibodies directed against AIC components in immunofluorescence microscopy experiments to visualize the recruitment of AIC components to forming phagosomes that contained beads. We found robust recruitment of AMPK $\alpha$ and AIC component ULK1 to forming phagosomes containing HA-coated beads (Figure 3E-H). We also incubated HA-coated beads with GFP-Gal8 expressing macrophages and found that recruitment of AMPK $\alpha$ or galectin 8 to the sites of bead internalization was not detected (Figure S3B, upper). However, co-localization of CD44 and AMPK $\alpha$ was observed to be colocalized with the HA-coated beads (Figure S3B, lower), Our observations therefore suggested that HA interactions with CD44 were necessary and sufficient to induce the formation of an AIC protein complex on forming phagosomes.

\section{Interaction of fungal HA with host CD44 activates AMPK and AIC pathways}

23 The observation that interactions between HA and host CD44 recruited AIC components to

24 forming phagosomes raised questions about the mechanism of AIC recruitment by these 25 components. HA induces $\mathrm{Ca}^{2+}$ elevation in cells and may increase the activity of $\mathrm{Ca}^{2+}$-associated 26 signaling pathways (Singleton and Bourguignon, 2002). For example, an increase of cytoplasmic

$27 \mathrm{Ca}^{2+}$ levels can induce autophagy through CaMKK $\beta$ and AMPK pathways (Feng et al., 2020; 28 Green et al., 2011). To test whether HA and CD44 interactions elevate intracellular $\mathrm{Ca}^{2+}\left(\left[\mathrm{Ca}^{2+}\right]_{\mathrm{i}}\right)$ 29 levels and induce autophagy by $\mathrm{Ca}^{2+}$-mediated activation of $\mathrm{CaMKK} \beta$ and AMPK pathways, we 30 incubated host cells with Cn cells, HA, naked beads, or HA-coated beads, and then visualized

$31\left[\mathrm{Ca}^{2+}\right]_{\mathrm{i}}$ levels and activation of CaMKK $\beta$-AMPK pathways in the treated cells. We observed that 
1 compared to the controls, $c p s 1^{+-} \mathrm{Cn}$ induced $\mathrm{Ca}^{2+}$-fluxes and increased $\left[\mathrm{Ca}^{2+}\right]_{\mathrm{i}}$ concentrations in a

2 pulsed manner (Figure 4A-C; Videos 1-2). Similar results were observed in cells incubated with

3 HA or HA-coated beads (Figure S3C), which is consistent with results from a previous report

4 (Singleton and Bourguignon, 2002). Corresponding to the increase of $\left[\mathrm{Ca}^{2+}\right]_{\mathrm{i}}$ levels, activation of 5 CaMKK $\beta$ and AMPK $\alpha$ was observed in H99 cells, (Pandey et al., 2017), or HA or HA-coated

6 beads (Figure 4D-E). Importantly, phosphorylated AMPK $\alpha$ and ATG9 were co7 immunoprecipitated from host cells incubated with HA-coated, but not naked, beads (Figure 4F).

9 To test whether activation of AMPK resulted from the increase of $\left[\mathrm{Ca}^{2+}\right]_{\mathrm{i}}$, we treated host cells 10 with or without supplementation of assorted inhibitors, including BAPTA-AM (a $\left[\mathrm{Ca}^{2+}\right]_{\mathrm{i}}$-chelator), 11 KN62 (a specific CaMK inhibitor), and STO-609 (a specific CaMKK $\alpha / \beta$ inhibitor), during 12 infection with $\mathrm{Cn}$. We found that host cells treated with these compounds displayed reduced 13 activation of AMPK (Figure 4G); the activation of the downstream target ULK1 was also almost 14 completely blocked (Figure 4H). During $\mathrm{Cn}$ infection, ULK1 is activated in a p-AMPK15 dependent fashion (Figure 4G-I). Consequently, $\mathrm{Cn}$ internalization was reduced in cells in which $16\left[\mathrm{Ca}^{2+}\right]_{\mathrm{i}}$ was chelated, the activities of $\mathrm{CaMK}$ or $\mathrm{CaMKK} \alpha / \beta$ were inhibited or depleted (Figure 4J;

17 Figure S4A-D), or AMPK or AIC components were depleted (Figure S4E-F). These findings 18 suggest that interaction of HA with CD44 recruits AIC to forming phagosomes by release of $\mathrm{Ca}^{2+}$ 19 and activation of the CaMKK $\beta$-AMPK-ULK1 signaling axis.

\section{Discussion}

The intracellular lifestyle of $\mathrm{Cn}$ is pivotal for pathogen colonization, dissemination and disease

24 progression (Garcia-Rodas and Zaragoza, 2012; Johnston and May, 2013; Seider et al., 2010), as

25 well as establishment of latent infection (Saha et al., 2007). Although the complete set of host

26 factors that regulate intracellular parasitism remain obscure, published reports demonstrate that

27 both "zipper" (receptor-mediated) and "trigger" (membrane ruffle dependent) mechanisms

28 contribute to the internalization of Cn (Guerra et al., 2014). Moreover, interactions between the

29 opsonized or unopsonized pathogen and host cell surface receptors are important to these

30 processes (Shoham et al., 2001; Taborda and Casadevall, 2002). The findings reported here

31 provide a new understanding of phagocytic mechanisms by demonstrating that fungal HA- and 
1 host CD44-dependent recruitment of AIC network components to nascent CnCVs play a central 2 role in regulating the internalization of the fungus. Cn cells, opsonized or unopsonized and live or 3 dead, as well as HA-coated beads, efficiently recruited host CD44, components of AIC and its

4 regulatory protein AMPK to forming or formed phagosomes. As such, this report provides the first 5 example of ligand- and receptor-induced recruitment of AIC proteins, including ULK1, FIP200, ATG13, and ATG9, to nascent phagosomes in macrophages.

8 Host cell galectin 8 is involved in defending against bacterial infection by recruiting the autophagic adaptor NDP52 to damaged Salmonella-containing vacuoles and in activating antibacterial autophagy (Thurston et al., 2012). Different from the defensive autophagy induced by

11 galectin 8, significant recruitment of the danger receptor galectin 8 to forming or formed 12 phagosomes during $\mathrm{Cn}$ internalization and/or intracellular replication was not observed, 13 suggesting that a different strategy is employed to recruit elements of the autophagy machinery 14 through interactions between fungal HA and host cell CD44. Besides AMPK and AIC components, 15 the endosomal and lysosomal markers EEA1, M6PR, and Cathepsin D, as well as the ER marker 16 calreticulin, were recruited to nascent phagosomes (Qin et al., 2011). Interestingly, co-localization 17 of the endocytic marker EEA1 and the AIC component ULK1 was also observed, suggesting that 18 the endosomal and lysosomal pathways, ER-derived membrane and selective autophagy 19 machinery are involved in the internalization of $\mathrm{Cn}$ into host cells. How these factors coordinate 20 this process and the involvement of other ubiquitin-binding autophagic adaptors related to the AIC 21 remain to be characterized.

AMPK can be activated by cellular stresses that elevate AMP levels by means of allosteric binding of AMP to sites in the $\gamma$ subunit AMPK, and by phosphorylation of Thr172 in AMPK $\alpha$ by the tumor suppressor LKB1, CaMKK $\beta$, or the transforming growth factor- $\beta$-activated kinase (TAK1) (Hardie et al., 2012; Kola et al., 2006; Zadra et al., 2015). Activation of AMPK can induce autophagy via direct phosphorylation of ULK1 (Egan et al., 2011; Kim et al., 2011; Zhao and

28 Klionsky, 2011). Previous observations showed that HA interactions with CD44 induces $\mathrm{Ca}^{2+}$ 29 elevation in cells (Singleton and Bourguignon, 2002), and that increases in cytoplasmic $\mathrm{Ca}^{2+}$ 30 concentrations induce autophagy through activation of CaMKK $\beta$ and AMPK pathways (Feng et 31 al., 2020; Green et al., 2011). Similarly, our findings demonstrate that infection by $\mathrm{Cn}$ recruits 
1 host cell $\mathrm{CD} 44$ to $\mathrm{CnCVs}$, induces intracellular $\mathrm{Ca}^{2+}$-flux in the infected host cells, activates

$2 \mathrm{CaMKK} \beta$ and $\mathrm{AMPK}$, and recruits AIC components to forming or nascent CnCVs. Cn infection

3 also activates LKB1, and depletion of LKB1 reduced Cn internalization into host cells (Pandey et

4 al., 2017). However, how the interaction of HA and CD44 activates AMPK through the upstream

5 regulatory proteins $\mathrm{LKB} 1$ and TAK1 remains to be further characterized.

7 Our data support a stepwise model in which several sequential molecular events control the 8 internalization of $\mathrm{Cn}$ in macrophages. First, interactions between fungal HA and CD44 on the 9 surface of host cells stimulates the release of $\mathrm{Ca}^{2+}$ and elevates intercellular $\mathrm{Ca}^{2+}$ levels, which

10 results in the activation of CaMKK $\beta$ and AMPK. Second, phosphorylation of ULK1 occurs in an 11 AMPK-dependent fashion. Third, AMPK-dependent activation of ULK1 recruits AIC components, 12 including the ULK1-ATG13-FIP200 complex, ATG9, and LC3, to forming phagocytic cups 13 containing the fungus. Fourth, the coordinated activities of AIC components drive the 14 internalization of the pathogen into host cells. Finally, AIC component interactions with CnCVs 15 gradually diminish as the pathogen establishes a replicative niche in host cells (Figure for 16 graphical Abstract).

18 Finally, it is notable that several proteins in AMPK $\alpha$ and AIC regulatory networks are targets of 19 commonly prescribed drugs (e.g., AMPK, a target for metformin) or under development as targets 20 for pharmaceutical intervention (e.g., ULK1) (Egan et al., 2015). Several fungi, including Candida 21 spp. and Histoplasma capsulatum, are capable of intracellular parasitism (Garcia-Rodas et al., 22 2011; Howard, 1965; Woods, 2003). Therefore, our findings may open up new therapeutic 23 possibilities for preventing cryptococcosis and other infections caused by intracellular pathogens.

\section{Acknowledgement}

26 We gratefully thank Arturo Casadevall (Department of Microbiology and Immunology, Albert

27 Einstein College of Medicine, Yeshiva University) for anti-cryptococcal antibodies, and Steve 28 Fullwood and Kalli Landua (Nikon Instruments) for expert assistance with the microscopy 29 analysis. This work is supported by the Texas AM Clinical Science Translational Research 30 Institute Pilot Grant CSTR2016-1, DARPA (HR001118A0025-FoF-FP-006), NIH 31 (R21AI139738-01A1, 1 R01AI141607-01A1, 1R21GM132705-01), the National Science 
Foundation (DBI 1532188, NSF0854684) and the Bill Melinda Gates Foundation, the Defense Advanced Research Projects Agency (Agreement HR001118A0025-FoF-FP-006) to PdF; NIH grant awards NIH 1R01 AI48496-01A1 and NIH 1U54AI057156-0100 to TAF; the National Natural Science Foundation of China (\# 81371773) to QMQ. Any opinions, findings, and conclusions or recommendations expressed in this material are those of the author(s) and do not necessarily reflect the views of the funding agencies.

\section{Author Contributions}

P.D., Q.M.Q., T.A.F. designed the experiments. S.L.D., A.P., X.H.F., Q.M.Q, J.Y., L.F.d.C., R.M.

S.L.B. performed experiments. P.D., T.A.F., A.R., R.M., R.O.W., K.P. provided reagents/analysis tools. P.D., Q.M.Q., S.L.D., X.H.F., A.P., T.A.F. analyzed data. P.D., Q.M.Q. supervised the work and wrote the manuscript.

\section{References}

Abada, A., and Elazar, Z. (2014). Getting ready for building: signaling and autophagosome biogenesis. EMBO Rep.

Campeau, E., Ruhl, V.E., Rodier, F., Smith, C.L., Rahmberg, B.L., Fuss, J.O., Campisi, J., Yaswen, P., Cooper, P.K., and Kaufman, P.D. (2009). A versatile viral system for expression and depletion of proteins in mammalian cells. PloS one 4.

Case, E.D.R., Smith, J.A., Ficht, T.A., Samuel, J.E., and de Figueiredo, P. (2016). Space: a final frontier for vacuolar pathogens. Traffic 17, 461-474.

de Figueiredo, P., and Dickman, M. (2016). Plant Disease: Autophagy under attack. eLife 5, e14447.

de Figueiredo, P., Ficht, T.A., Rice-Ficht, A., Rossetti, C.A., and Adams, L.G. (2015). Pathogenesis and immunobiology of brucellosis: review of Brucella-Host Interactions. The American journal of pathology 185, 1505-1517.

Dickman, M., Williams, B., Li, Y., de Figueiredo, P., and Wolpert, T. (2017). Reassessing apoptosis in plants. Nature plants 3, 773-779.

Egan, D.F., Chun, M.G., Vamos, M., Zou, H., Rong, J., Miller, C.J., Lou, H.J., Raveendra-Panickar, D., Yang, C.C., Sheffler, D.J., et al. (2015). Small Molecule Inhibition of the Autophagy Kinase ULK1 and Identification of ULK1 Substrates. Mol Cell 59, 285-297.

Egan, D.F., Shackelford, D.B., Mihaylova, M.M., Gelino, S., Kohnz, R.A., Mair, W., Vasquez, D.S., Joshi, A., Gwinn, D.M., and Taylor, R. (2011). Phosphorylation of ULK1 (hATG1) by AMP-activated protein kinase connects energy sensing to mitophagy. Science 331, 456-461.

Evans, R.J., Sundaramurthy, V., and Frickel, E.-M. (2018). The interplay of host autophagy and eukaryotic pathogens. Frontiers in Cell and Developmental Biology 6, 118.

Feng, N., Wang, B., Cai, P., Zheng, W., Zou, H., Gu, J., Yuan, Y., Liu, X., Liu, Z., and Bian, J. (2020). ZEAinduced autophagy in TM4 cells was mediated by the release of Ca2+ activates CaMKK $\beta$-AMPK signaling pathway in the endoplasmic reticulum. Toxicology Letters.

Galluzzi, L., Baehrecke, E.H., Ballabio, A., Boya, P., Bravo-San Pedro, J.M., Cecconi, F., Choi, A.M., Chu, C.T., Codogno, P., and Colombo, M.I. (2017). Molecular definitions of autophagy and related processes. The EMBO journal 36, 1811-1836. 
Ganley, I.G., Lam, D.H., Wang, J., Ding, X., Chen, S., and Jiang, X. (2009). ULK1· ATG13· FIP200 complex mediates mTOR signaling and is essential for autophagy. Journal of Biological Chemistry 284, 12297-12305.

Garcia-Rivera, J., Chang, Y.C., Kwon-Chung, K.J., and Casadevall, A. (2004). Cryptococcus neoformans CAP59 (or Cap59p) is involved in the extracellular trafficking of capsular glucuronoxylomannan. Eukaryot Cell 3, 385-392.

Garcia-Rodas, R., Gonzalez-Camacho, F., Rodriguez-Tudela, J.L., Cuenca-Estrella, M., and Zaragoza, O. (2011). The interaction between Candida krusei and murine macrophages results in multiple outcomes, including intracellular survival and escape from killing. Infect Immun 79, 2136-2144.

Garcia-Rodas, R., and Zaragoza, O. (2012). Catch me if you can: phagocytosis and killing avoidance by Cryptococcus neoformans. FEMS Immunol Med Microbiol 64, 147-161.

Green, M.F., Anderson, K.A., and Means, A.R. (2011). Characterization of the CaMKK $\beta$-AMPK signaling complex. Cellular signalling 23, 2005-2012.

Guerra, C.R., Seabra, S.H., de Souza, W., and Rozental, S. (2014). Cryptococcus neoformans is internalized by receptor-mediated or 'triggered' phagocytosis, dependent on actin recruitment. PLoS One 9, e89250.

Hardie, D.G., Ross, F.A., and Hawley, S.A. (2012). AMPK: a nutrient and energy sensor that maintains energy homeostasis. Nature reviews Molecular cell biology 13, 251-262.

Hosokawa, N., Hara, T., Kaizuka, T., Kishi, C., Takamura, A., Miura, Y., Iemura, S.-i., Natsume, T., Takehana, K., and Yamada, N. (2009). Nutrient-dependent mTORC1 association with the ULK1-Atg13-FIP200 complex required for autophagy. Molecular biology of the cell 20,1981-1991.

Howard, D.H. (1965). Intracellular Growth of Histoplasma Capsulatum. J Bacteriol 89, 518-523.

Irving, A.T., Mimuro, H., Kufer, T.A., Lo, C., Wheeler, R., Turner, L.J., Thomas, B.J., Malosse, C., Gantier, M.P., Casillas, L.N., et al. (2014). The immune receptor NOD1 and kinase RIP2 interact with bacterial peptidoglycan on early endosomes to promote autophagy and inflammatory signaling. Cell host \& microbe $15,623-635$.

Johnston, S.A., and May, R.C. (2010). The human fungal pathogen Cryptococcus neoformans escapes macrophages by a phagosome emptying mechanism that is inhibited by Arp2/3 complex-mediated actin polymerisation. PLoS Pathog 6, e1001041.

Johnston, S.A., and May, R.C. (2013). Cryptococcus interactions with macrophages: evasion and manipulation of the phagosome by a fungal pathogen. Cell Microbiol 15, 403-411.

Jong, A., Wu, C.H., Chen, H.M., Luo, F., Kwon-Chung, K.J., Chang, Y.C., Lamunyon, C.W., Plaas, A., and Huang, S.H. (2007). Identification and characterization of CPS1 as a hyaluronic acid synthase contributing to the pathogenesis of Cryptococcus neoformans infection. Eukaryot Cell 6, 1486-1496.

Jong, A., Wu, C.H., Gonzales-Gomez, I., Kwon-Chung, K.J., Chang, Y.C., Tseng, H.K., Cho, W.L., and Huang, S.H. (2012). Hyaluronic acid receptor CD44 deficiency is associated with decreased Cryptococcus neoformans brain infection. J Biol Chem 287, 15298-15306.

Jong, A., Wu, C.H., Shackleford, G.M., Kwon-Chung, K.J., Chang, Y.C., Chen, H.M., Ouyang, Y., and Huang, S.H. (2008). Involvement of human CD44 during Cryptococcus neoformans infection of brain microvascular endothelial cells. Cellular microbiology 10, 1313-1326.

Jung, C.H., Jun, C.B., Ro, S.-H., Kim, Y.-M., Otto, N.M., Cao, J., Kundu, M., and Kim, D.-H. (2009). ULKAtg13-FIP200 complexes mediate mTOR signaling to the autophagy machinery. Molecular biology of the cell 20, 1992-2003.

Khandia, R., Dadar, M., Munjal, A., Dhama, K., Karthik, K., Tiwari, R., Yatoo, M., Iqbal, H., Singh, K.P., and Joshi, S.K. (2019). A comprehensive review of autophagy and its various roles in infectious, non-infectious, and lifestyle diseases: current knowledge and prospects for disease prevention, novel drug design, and therapy. Cells 8,674.

Kim, J., Kundu, M., Viollet, B., and Guan, K.-L. (2011). AMPK and mTOR regulate autophagy through direct phosphorylation of Ulk1. Nature cell biology 13, 132-141.

Kirkin, V., and Rogov, V.V. (2019). A Diversity of Selective Autophagy Receptors Determines the Specificity of the Autophagy Pathway. Molecular cell.

Kola, B., Boscaro, M., Rutter, G.A., Grossman, A.B., and Korbonits, M. (2006). Expanding role of AMPK in endocrinology. Trends in Endocrinology \& Metabolism 17, 205-215.

Kozubowski, L., and Heitman, J. (2012). Profiling a killer, the development of Cryptococcus neoformans. FEMS Microbiol Rev 36, 78-94. 
Mack, H.I., Zheng, B., Asara, J.M., and Thomas, S.M. (2012). AMPK-dependent phosphorylation of ULK1 regulates ATG9 localization. Autophagy 8, 1197-1214.

Nicola, A.M., Albuquerque, P., Martinez, L.R., Dal-Rosso, R.A., Saylor, C., De Jesus, M., Nosanchuk, J.D., and Casadevall, A. (2012). Macrophage autophagy in immunity to Cryptococcus neoformans and Candida albicans. Infect Immun 80, 3065-3076.

O'Meara, T.R., and Alspaugh, J.A. (2012). The Cryptococcus neoformans capsule: a sword and a shield. Clin Microbiol Rev 25, 387-408.

Olszewski, M.A., Zhang, Y., and Huffnagle, G.B. (2010). Mechanisms of cryptococcal virulence and persistence. Future Microbiol 5, 1269-1288.

Pandey, A., Ding, S.L., Qin, Q.M., Gupta, R., Gomez, G., Lin, F., Feng, X., Fachini da Costa, L., Chaki, S.P., Katepalli, M., et al. (2017). Global Reprogramming of Host Kinase Signaling in Response to Fungal Infection. Cell host \& microbe 21,637-649 e636.

Pandey, A., Lin, F., Cabello, A.L., da Costa, L.F., Feng, X., Feng, H.-Q., Zhang, M.-Z., Iwawaki, T., Rice-Ficht, A., and Ficht, T.A. (2018). Activation of host IRE1 $\alpha$-dependent signaling axis contributes the intracellular parasitism of Brucella melitensis. Frontiers in Cellular and Infection Microbiology 8, 103.

Qin, Q.M., Luo, J., Lin, X., Pei, J., Li, L., Ficht, T.A., and de Figueiredo, P. (2011). Functional analysis of host factors that mediate the intracellular lifestyle of Cryptococcus neoformans. PLoS Pathog 7, e1002078.

Rubinsztein, D.C., Shpilka, T., and Elazar, Z. (2012). Mechanisms of autophagosome biogenesis. Curr Biol 22, R29-34.

Sabiiti, W., and May, R.C. (2012). Mechanisms of infection by the human fungal pathogen Cryptococcus neoformans. Future Microbiol 7, 1297-1313.

Sag, D., Carling, D., Stout, R.D., and Suttles, J. (2008). Adenosine 5'-monophosphate-activated protein kinase promotes macrophage polarization to an anti-inflammatory functional phenotype. Journal of immunology $181,8633-8641$.

Saha, D.C., Goldman, D.L., Shao, X., Casadevall, A., Husain, S., Limaye, A.P., Lyon, M., Somani, J., Pursell, K., Pruett, T.L., et al. (2007). Serologic evidence for reactivation of cryptococcosis in solid-organ transplant recipients. Clin Vaccine Immunol 14, 1550-1554.

Seider, K., Heyken, A., Luttich, A., Miramon, P., and Hube, B. (2010). Interaction of pathogenic yeasts with phagocytes: survival, persistence and escape. Curr Opin Microbiol 13, 392-400.

Shoham, S., Huang, C., Chen, J.M., Golenbock, D.T., and Levitz, S.M. (2001). Toll-like receptor 4 mediates intracellular signaling without TNF-alpha release in response to Cryptococcus neoformans polysaccharide capsule. J Immunol 166, 4620-4626.

Singleton, P.A., and Bourguignon, L.Y. (2002). CD44v10 interaction with Rho-kinase (ROK) activates inositol 1, 4, 5-triphosphate (IP3) receptor-mediated $\mathrm{Ca} 2+$ signaling during hyaluronan (HA)-induced endothelial cell migration. Cell motility and the cytoskeleton 53, 293-316.

Taborda, C.P., and Casadevall, A. (2002). CR3 (CD11b/CD18) and CR4 (CD11c/CD18) are involved in complement-independent antibody-mediated phagocytosis of Cryptococcus neoformans. Immunity 16, 791802.

Thurston, T.L., Wandel, M.P., von Muhlinen, N., Foeglein, A., and Randow, F. (2012). Galectin 8 targets damaged vesicles for autophagy to defend cells against bacterial invasion. Nature 482, 414-418.

Warkentien, T., and Crum-Cianflone, N.F. (2010). An update on Cryptococcus among HIV-infected patients. Int J STD AIDS 21, 679-684.

Woods, J.P. (2003). Knocking on the right door and making a comfortable home: Histoplasma capsulatum intracellular pathogenesis. Curr Opin Microbiol 6, 327-331.

Yang, Y., Kitagaki, J., Dai, R.M., Tsai, Y.C., Lorick, K.L., Ludwig, R.L., Pierre, S.A., Jensen, J.P., Davydov, I.V., Oberoi, P., et al. (2007). Inhibitors of ubiquitin-activating enzyme (E1), a new class of potential cancer therapeutics. Cancer Res 67, 9472-9481.

Zadra, G., Batista, J.L., and Loda, M. (2015). Dissecting the dual role of AMPK in cancer: from experimental to human studies. Molecular cancer research 13, 1059-1072.

Zaragoza, O. (2019). Basic principles of the virulence of Cryptococcus. Virulence 10, 490-501.

Zhao, M., and Klionsky, D.J. (2011). AMPK-dependent phosphorylation of ULK1 induces autophagy. Cell metabolism 13, 119-120. 


\section{$1 \quad$ Figures and Figure Legends}

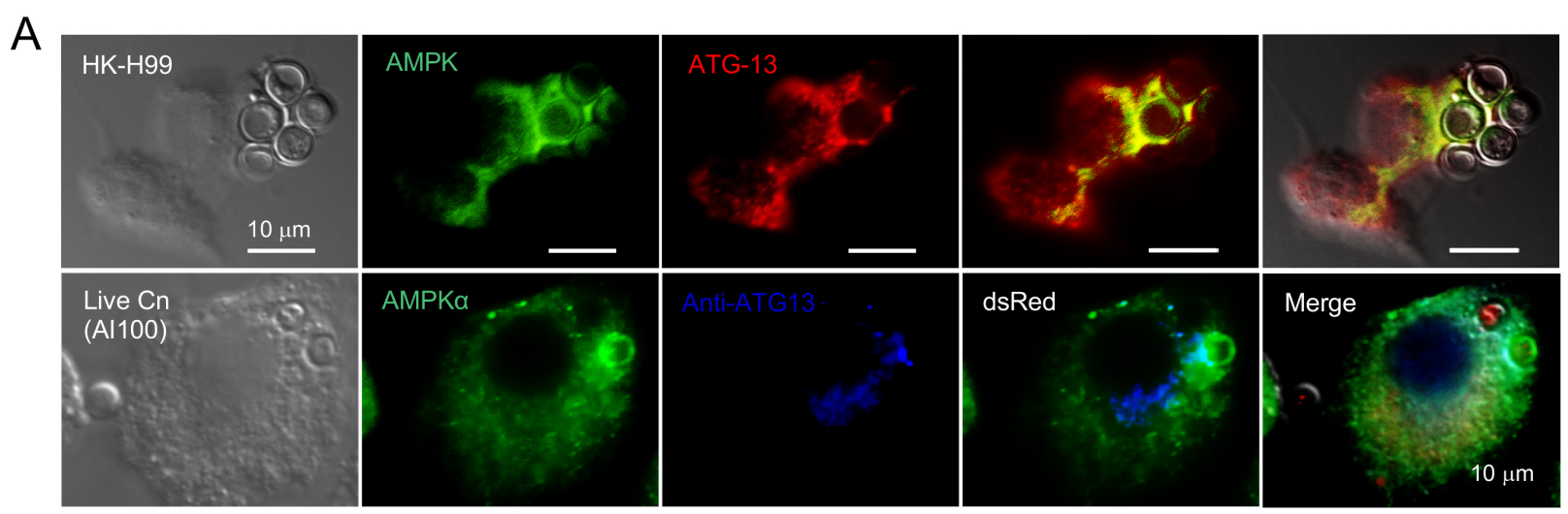

B

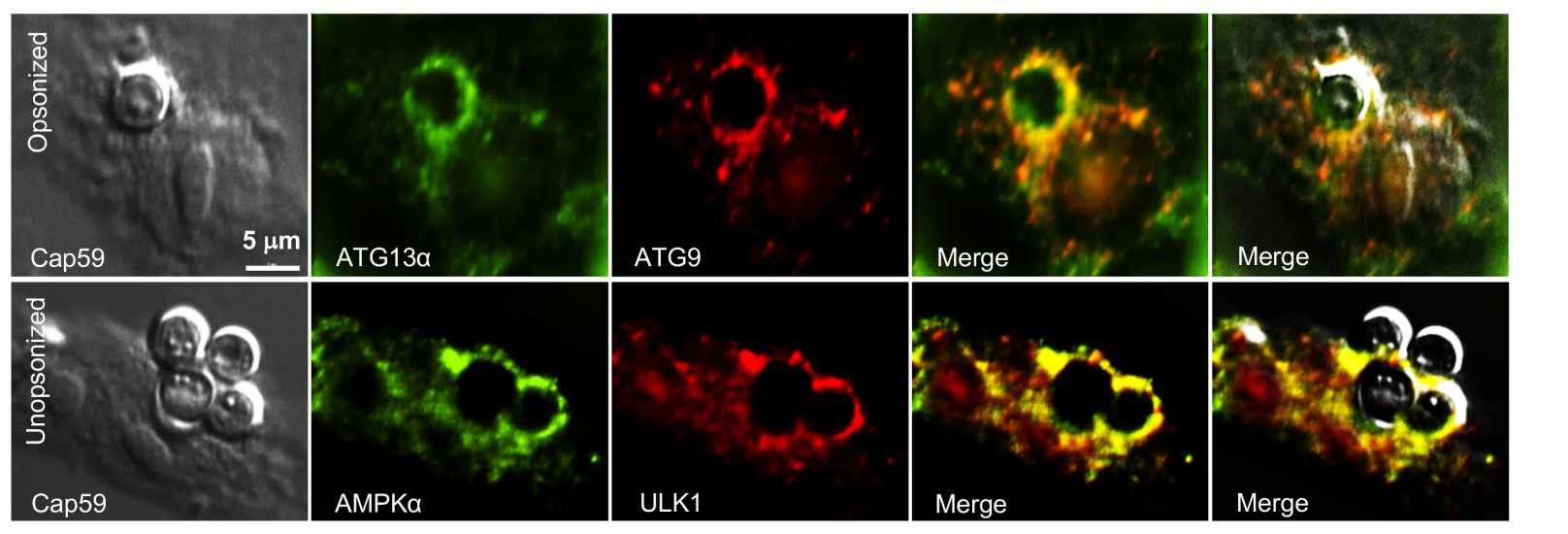

C
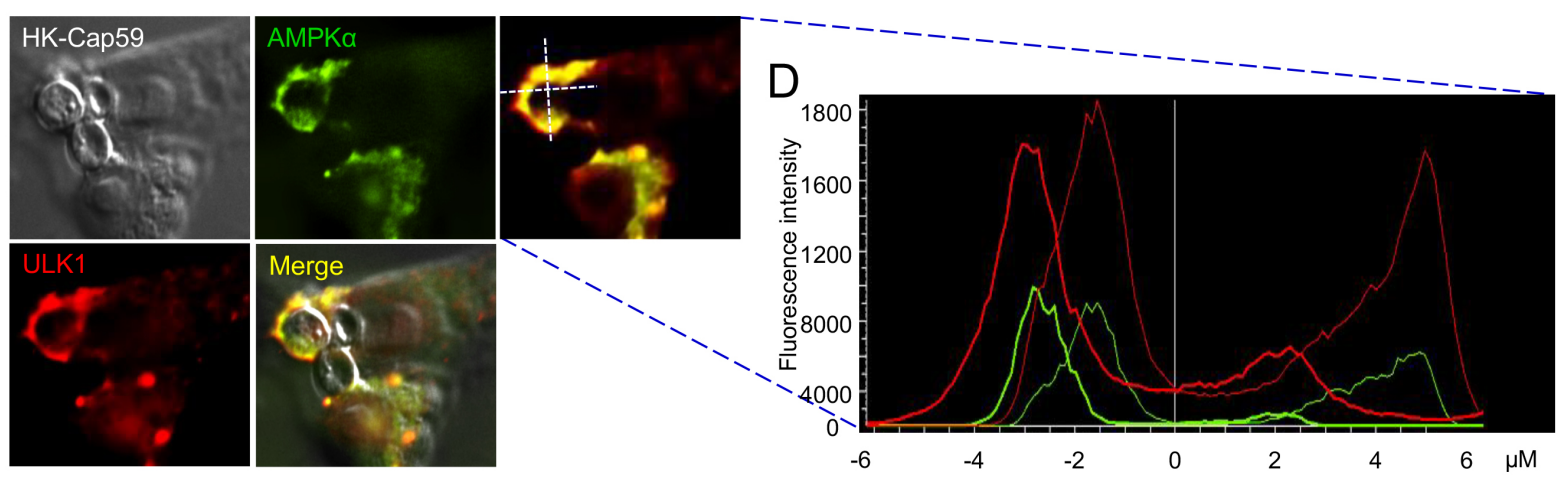

E

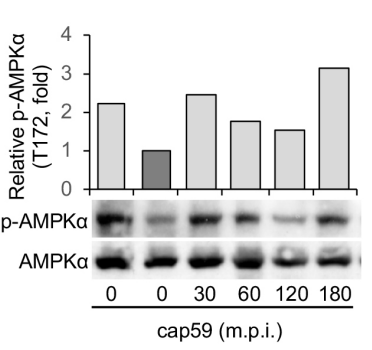

$\mathrm{F}$

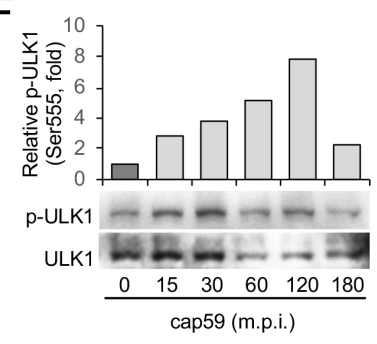

G

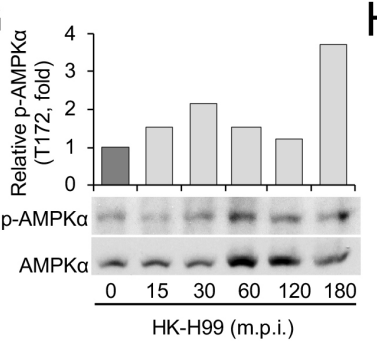
$\mathrm{H}$

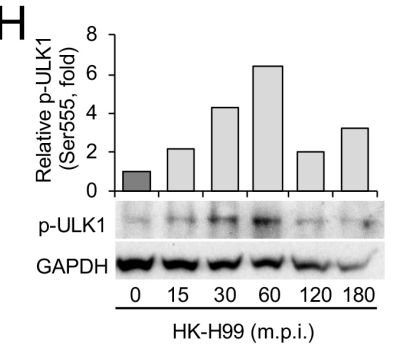


1 Figure 1. Non-proteineous components on $\mathrm{Cn}$ direct the recruitment of host AIC 2 components to nascent phagosomes.

3 (A, B) Colocalization of AMPK $\alpha$ and AIC components surrounding nascent CnCVs in host cells

4 infected with live or heat-killed (HK) H99 (A) and opsonized or unopsonized acapsular cap59

5 strains (B) of $\mathrm{Cn}$.

6 (C, D) Recruitment of AMPK $\alpha$ and ULK1 to nascent CnCVs in host cells incubated with HK-

7 cap59 at 3 h.p.i. (C) and the fluorescence intensity profile of AMPK $\alpha$ (green) and ULK1 (red)

8 along the two crossed white lines (D).

9 (E, F) Activation of host cell AMPK $\alpha$ (E) and ULK1 (F) by acapsular Cn strain cap59.

$10(\mathrm{G}, \mathrm{H})$ Activation of host cell AMPK $\alpha(\mathrm{G})$ and ULK1 $(\mathrm{H})$ by HK Cn strain H99 (HK-H99). 
A
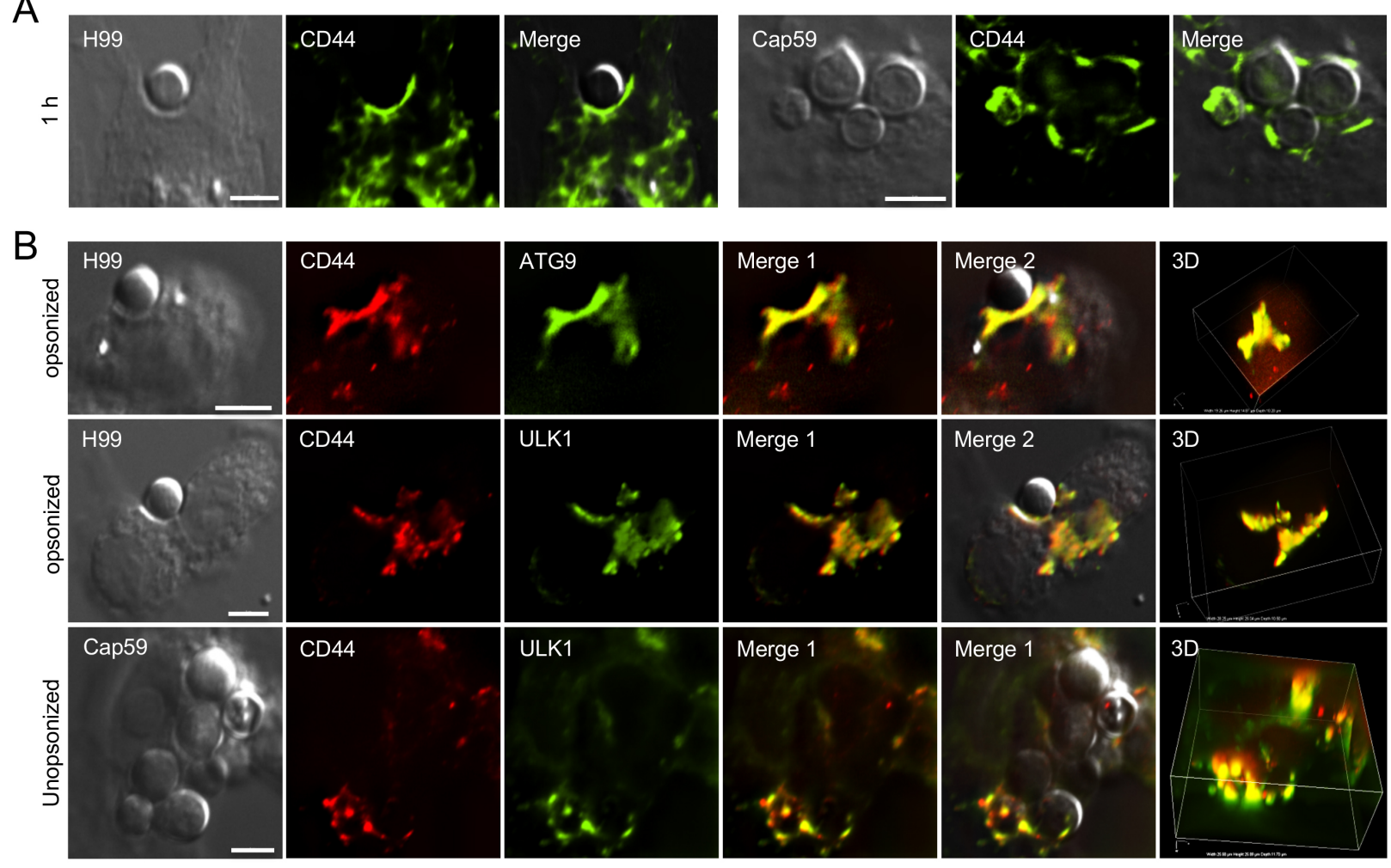

C

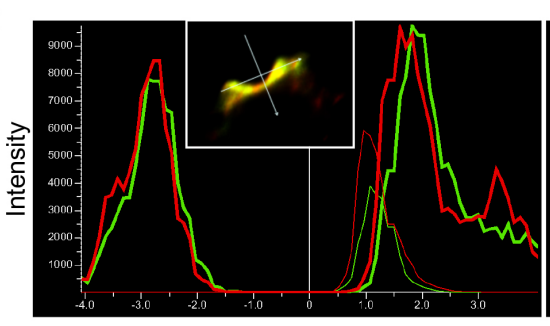

D

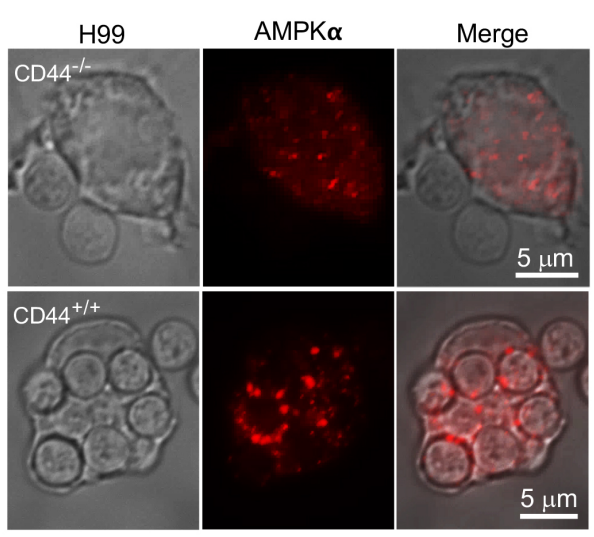

E
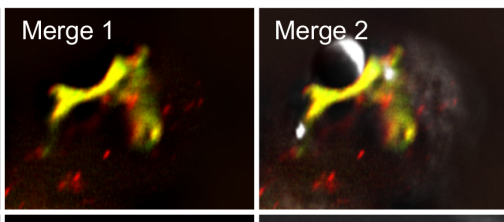

3D
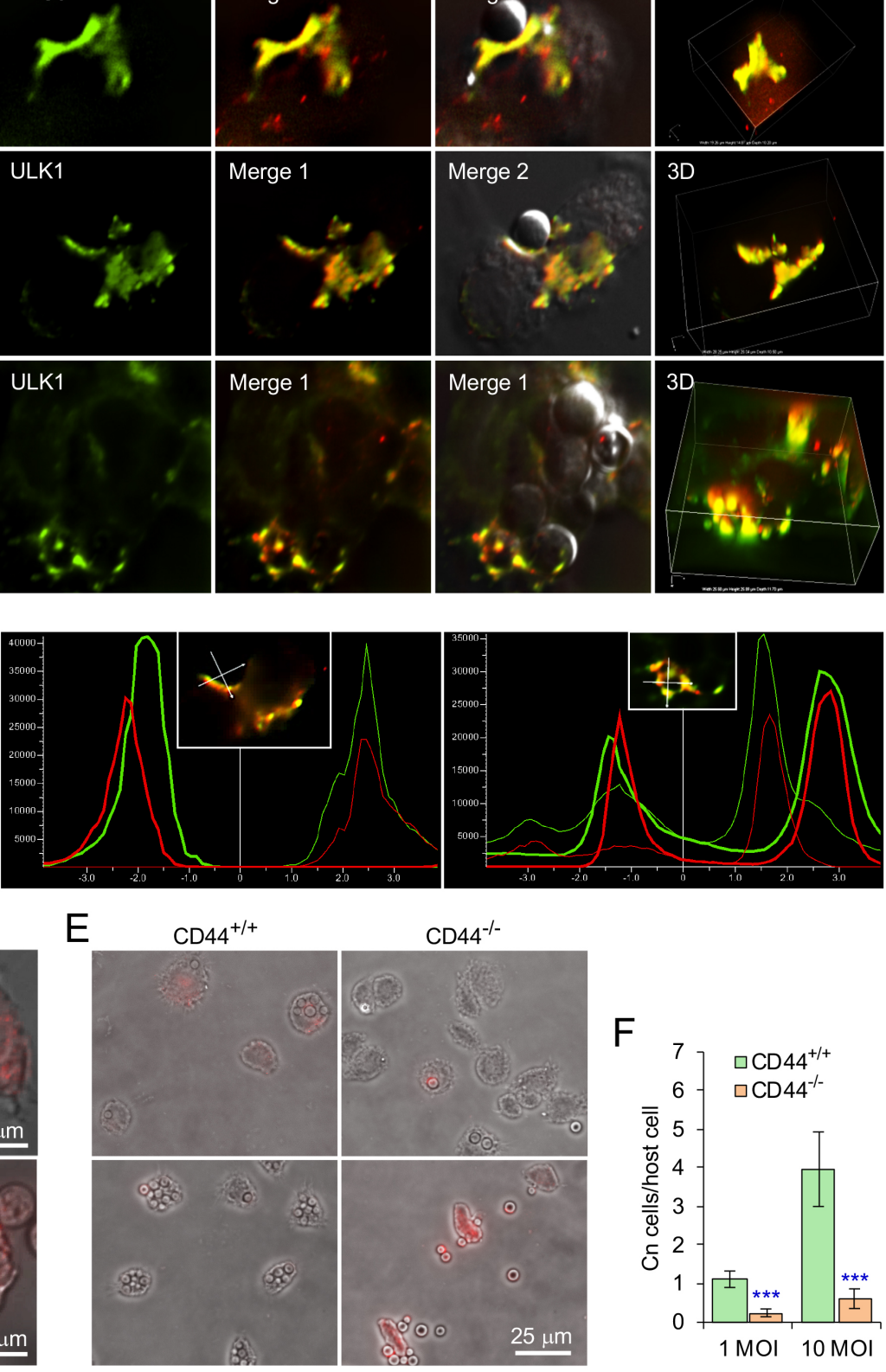
1 Figure 2. Host-associated CD44 is required for $\mathrm{Cn}$ host cell internalization.

2 (A) Recruitment of host CD44 to nascent CnCVs that contained the wild-type (WT) strain H99

3 (leaf panel) or acapsular mutant strain cap59 (right panel) of Cn. At $1 \mathrm{hr}$ post-infection (h.p.i.),

4 host cells were fixed, permeabilized and processed for immunofluorescence microscopy using

5 antibodies directed against the indicated host proteins. The antibody-stained samples were then

6 subjected to confocal microscopy image analysis.

7 (B) Colocalization of CD44 with the indicated AIC components in the vicinity of nascent CnCVs

8 in host cells infected with the indicated $\mathrm{Cn}$ strains.

9 (C) The fluorescence intensity profile of CD44 (red) and AIC components (ATG9 or ULK1)

10 (green) along the two crossed white lines shown in the insets from (B, Merge 1).

11 (D) AMPK recruitment to nascent CnCVs in CD44 knockout (KO, CD44-/) and WT $\left(\mathrm{CD}^{-/ 4} 4^{+/+}\right)$

12 bone marrow-derived macrophages (BMDMs) at 3 h.p.i..

13 (E, F) Cn internalization in CD44 WT and KO BMDMs infected by Cn H99 assessed using image

14 analysis approaches (E) and corresponding quantification (F) at 3 h.p.i.. Data represent the means

$15 \pm$ standard deviation (SD) from three independent experiments. $* * *$ : significance at $\mathrm{p}<0.001$. 


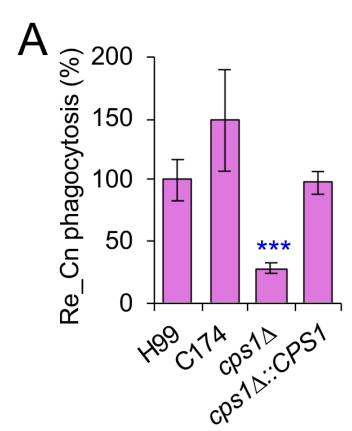

D
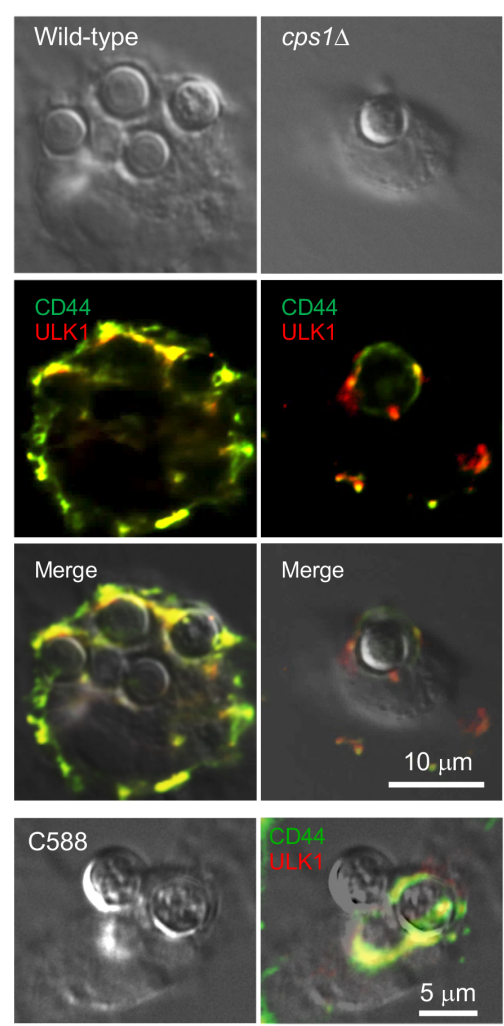

G

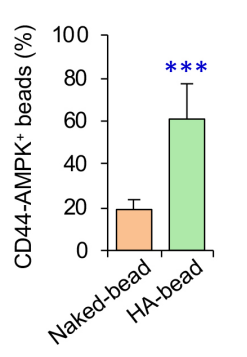

B

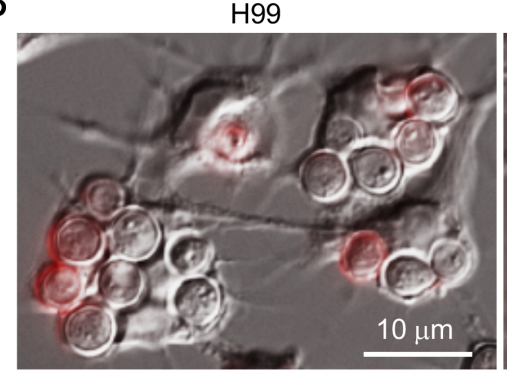

$\mathrm{E}$

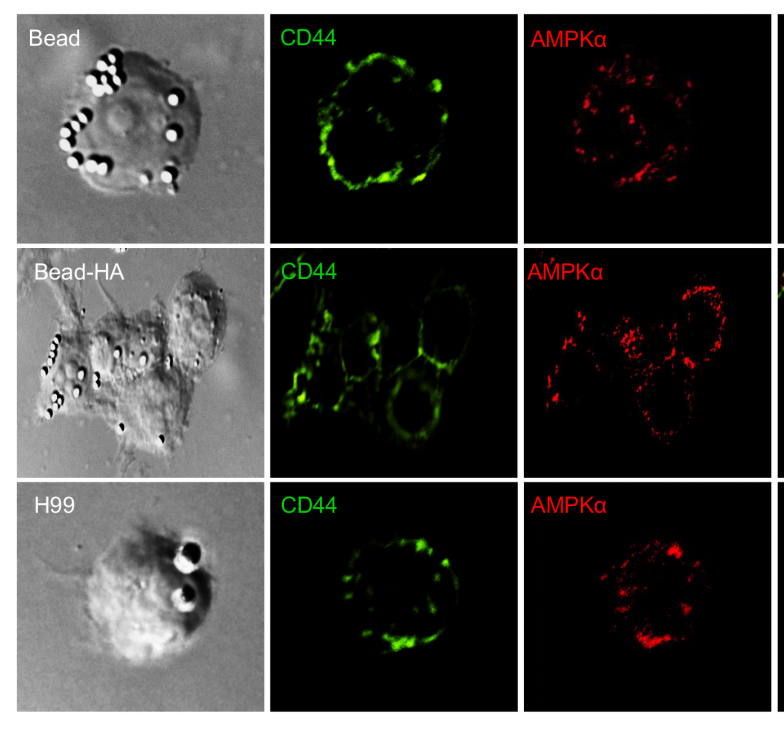

$\mathrm{F}$
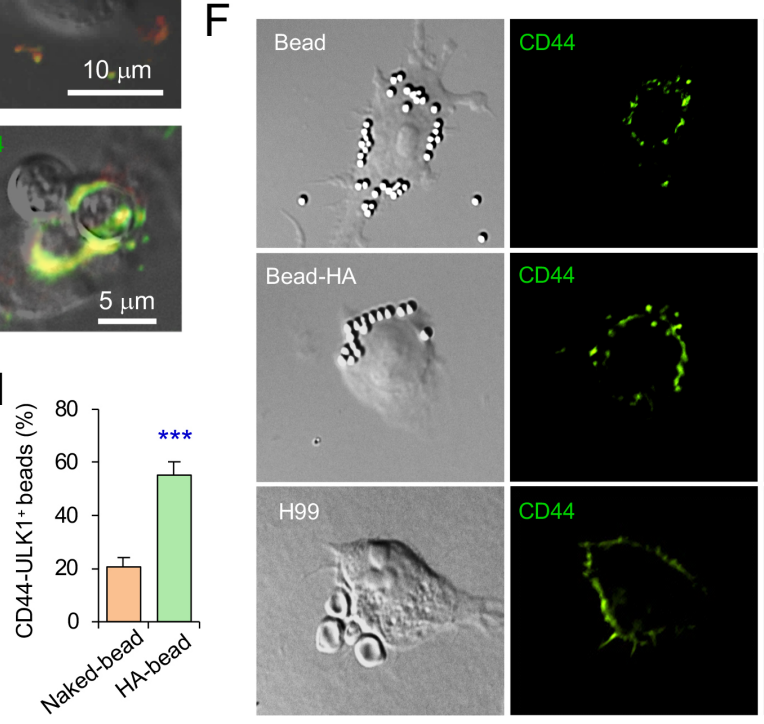

$\operatorname{cps} 1 \Delta$
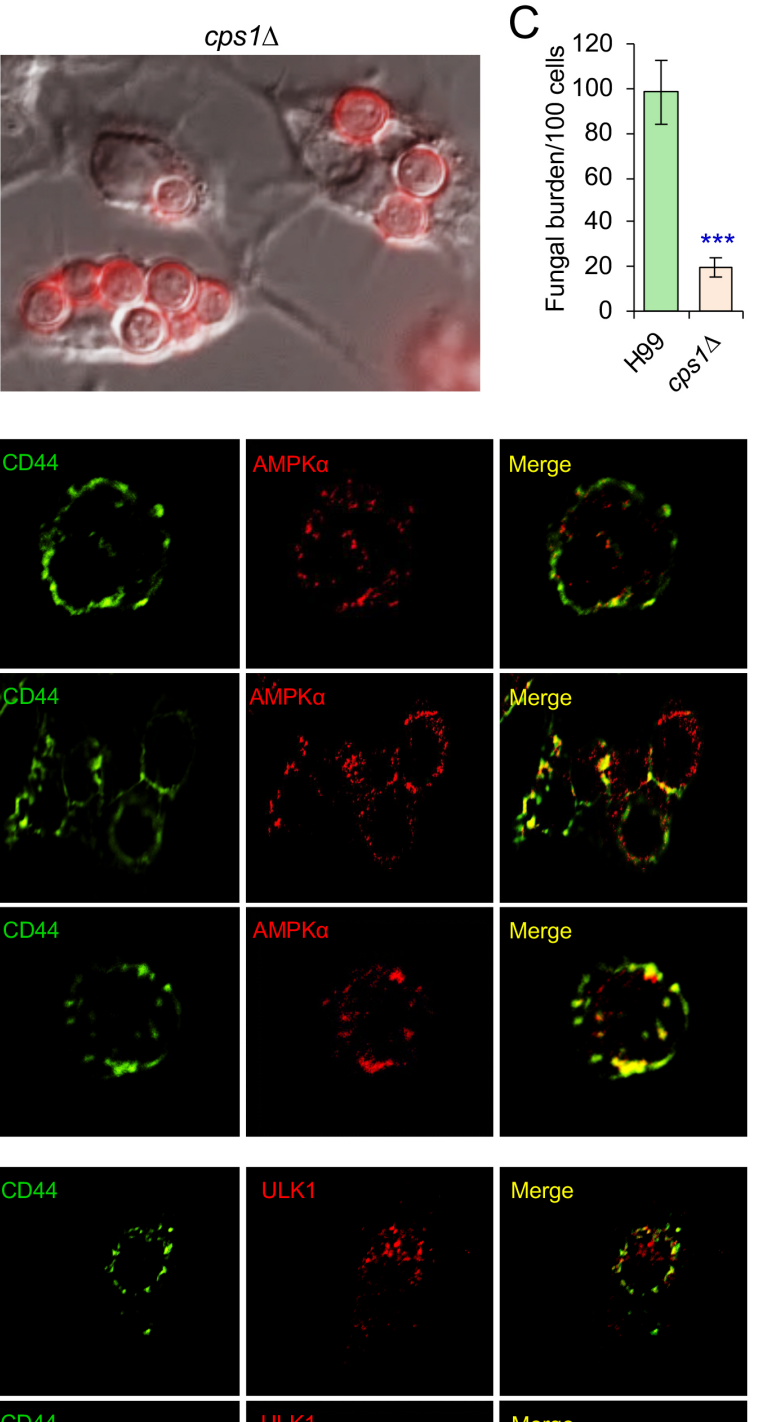

Merge
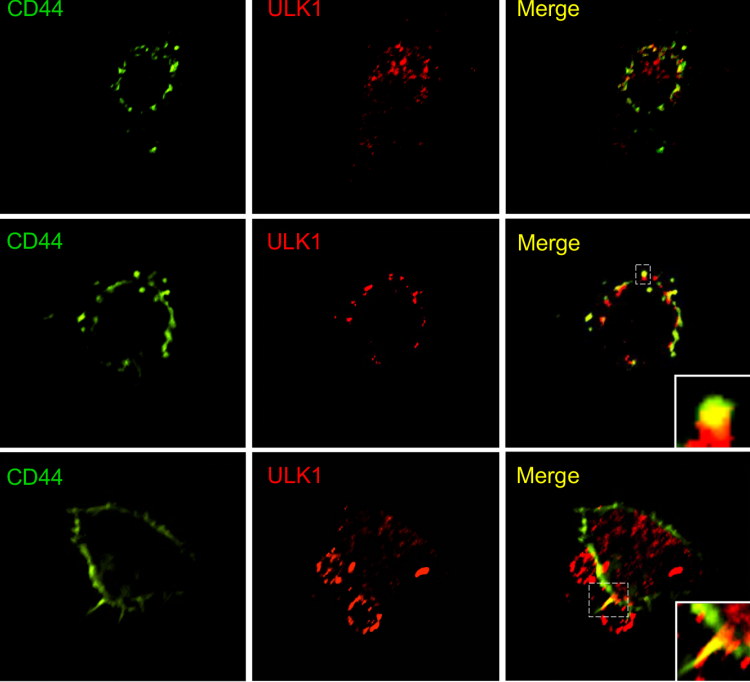
1 Figure 3. Fungal HA is required for recruitment of AMPK or AIC components to nascent 2 phagosomes and $\mathrm{Cn}$ internalization.

3 (A) Internalization of the wild-typ, $\operatorname{cps} 1 \Delta$ and complemented $\mathrm{Cn}$ strains.

4 (B-C) Internalization of the indicated Cn strains in BMDMs (B) and quantification of intracellular

5 Cn cells $(\mathrm{C})$ at 3 h.p.i..

6 (D) Confocal microscopy image analysis of recruitment of CD44 and the AIC component ULK1

7 by wild-type, $\operatorname{cps} 1 \Delta$, and complemented (C588) Cn strains at 3 h.p.i..

8 (E-F) Recruitment of host CD44 and AMPK $\alpha$ (E), or CD44 and AIC component ULK1 (F) to

9 nascent phagosomes or $\mathrm{CnCVs}$ by HA-coated beads or $\mathrm{Cn}$ (H99), respectively.

$10(\mathrm{G}, \mathrm{H})$ Quantification of CD44 and AMPK positive beads $(\mathrm{G})$ or CD44 and ULK1 positive beads

11 based on confocal microscopy images as shown in (E-F). Data represent the means \pm SD from

12 three independent experiments. $* * *$ : significance at $\mathrm{p}<0.001$.

13

14 

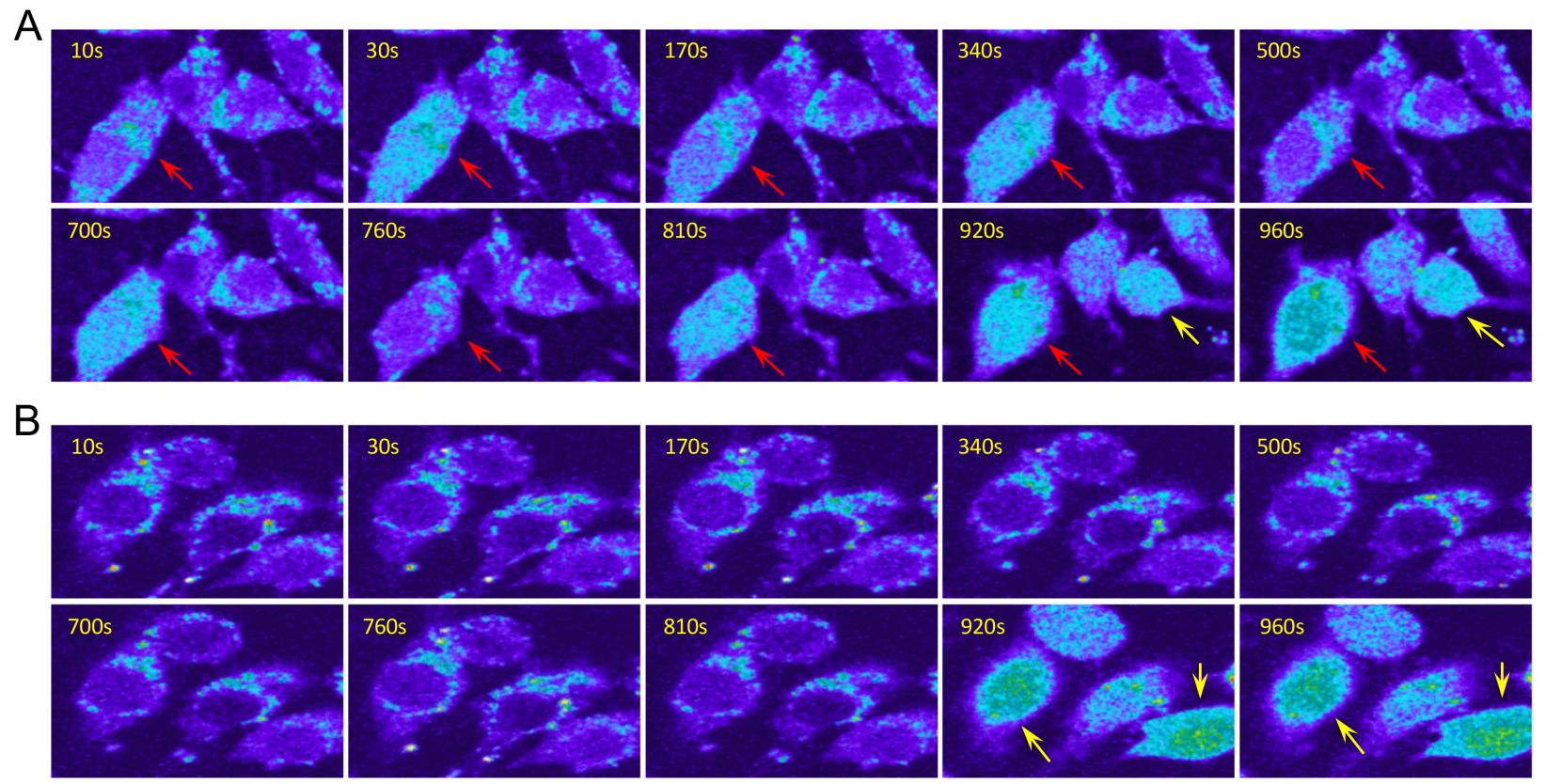

C

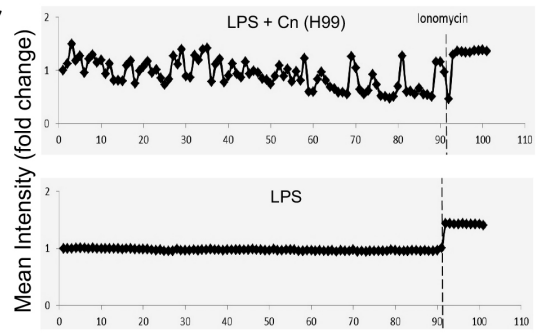

Time (X $10 \mathrm{sec}$ )

$\mathrm{F}$
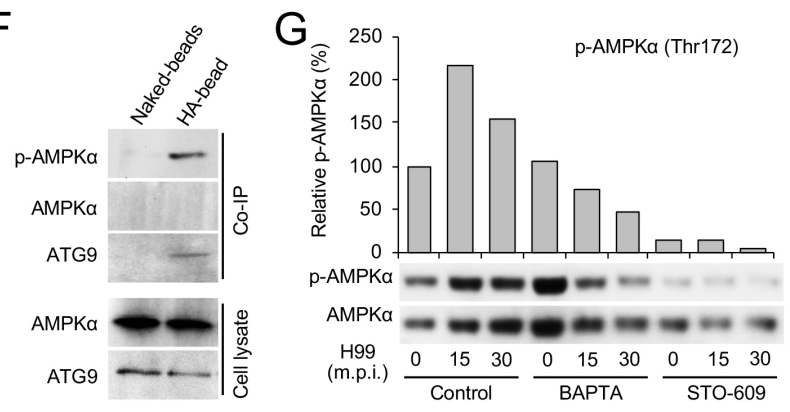

E

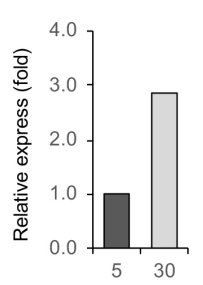

p-CamkK $1 \%$ GAPDH -

\section{$\mathrm{H}$}

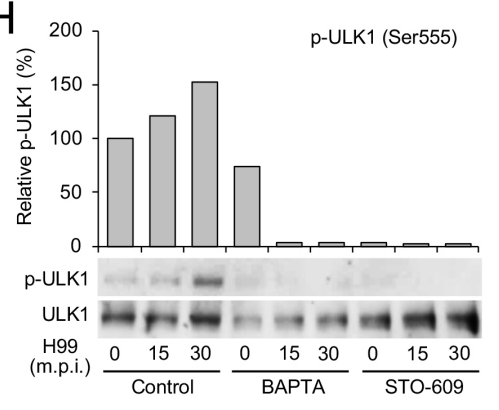

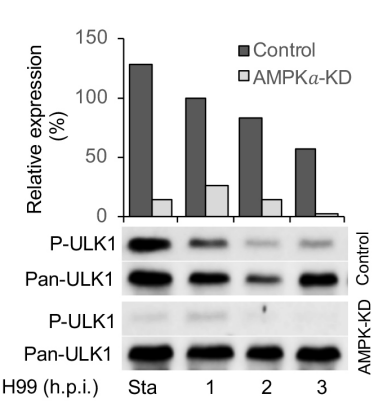

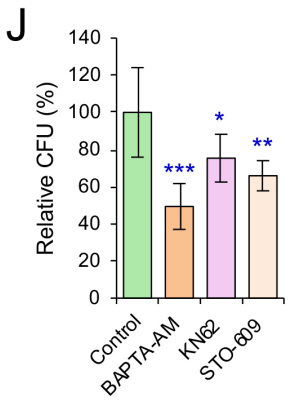




\section{Figure 4. Cn infection activates CaMKK $\beta$-AMPK-ULK1 signaling axis.}

2 (A, B) $\mathrm{Cn}$ infection results in intracellular $\mathrm{Ca}^{2+}$-flux in the infected host cells. Host cells incubated

3 with $\operatorname{cps}^{+} \mathrm{Cn}(\mathrm{A})$ or PBS (B) and the intracellular $\mathrm{Ca}^{2+}$ level was detected by excitation at $340 \mathrm{~nm}$.

4 Red arrows: $\mathrm{Ca}^{2+}$ releases from $\mathrm{cpsl}^{+}-\mathrm{Cn}$-infected host cells. Yellow arrows: $\mathrm{Ca}^{2+}$ releases from

5 cells treated with ionomycin, a membrane permeable calcium ionophore used to increase

6 intracellular calcium levels.

7 (C) Mean $\mathrm{Ca}^{2+}$ flux intensity of Cn-infected (upper panel) and control (lower panel) cells.

8 (D, E) Activation of host CaMKK $\beta$ (D) and AMPK $\alpha(\mathrm{E})$ in cells incubated with HA or HA-coated 9 beads.

10 (F) Co-immunoprecipitation (Co-IP) assays of host phosphorylated AMPK $\alpha$ and ATG9 trigged by

11 HA-coated beads. Host cells were incubated with Cn. At 3 h.p.i., the infected cells were lysed and 12 immunoprecipitated using antibodies against CD44. the precipitated material was then visualized 13 by Western blot analysis using antibodies directed against p-AMPK $\alpha$ or ATG9.

$14(\mathrm{G}, \mathrm{H})$ Chelation of intracellular $\mathrm{Ca}^{2+}$ by BAPTA-AM and inhibition of the activity of CaMKK $\beta$

15 by STO-609 reduce activation of AMPK $\alpha(\mathrm{G})$ and ULK1 $(\mathrm{H})$.

16 (I) Depletion of AMPK $\alpha$ reduces activation of ULK1 during Cn internalization. Sta: starvation.

17 (J) Cn internalization in host cells treated by the indicated drug via CFU assays. Data represent the

18 means \pm SD from three independent experiments. *, **, ***: significance at $\mathrm{p}<0.05,0.01$, and 190.001 , respectively. 


\section{Star Methods}

\section{KEY RESOURCES TABLE}

\begin{tabular}{|c|c|c|}
\hline REAGENT or RESOURCE & SOURCE & IDENTIFIER \\
\hline \multicolumn{3}{|l|}{ Antibodies } \\
\hline Mouse anti-C. neoformans 18B7 & $\begin{array}{l}\text { Dr. Arturo Casadevall, (Albert Einstein College of } \\
\text { Medicine of Yeshiva University, NY, USA) }\end{array}$ & N/A \\
\hline Mouse anti-HCAM(CD44) & Santa Cruz Biotechnology, Inc. (Dallas, TX USA) & Cat. \#: sc-7297 \\
\hline anti-pCaMKKß & Cell Signaling Technology, Inc. (Danver, MA USA) & Cat \#:16737S \\
\hline anti-CaMKKa & Santa Cruz Biotechnology, Inc. (Dallas, TX USA) & Cat \#: sc-11370/17827 \\
\hline anti-CaMKK $\beta$ & Santa Cruz Biotechnology, Inc. (Dallas, TX USA) & Cat. \#: sc-271674 \\
\hline Rabbit anti-pAMPKa (Thr 172) & Cell Signaling Technology, Inc. (Danver, MA USA) & Cat. \#: 2535 \\
\hline Rabbit anti-AMPKa & Cell Signaling Technology, Inc. (Danver, MA USA) & Cat. \#: 5831 \\
\hline Rabbit-anti pULK1 (Ser 555) & Cell Signaling Technology, Inc. (Danver, MA USA) & Cat. \#: 5869 \\
\hline Rabbit anti-ULK1 & Santa Cruz Biotechnology, Inc. (Dallas, TX USA) & Cat. \#: sc-33182 \\
\hline Rabbit anti-LC3 & Santa Cruz Biotechnology, Inc. (Dallas, TX USA) & Cat. \#: sc-134226 \\
\hline Rabbit anti-GAPDH & Santa Cruz Biotechnology, Inc. (Dallas, TX USA) & Cat. \#: sc-25778 \\
\hline Rabbit anti-ATG13 & Sigma-Aldrich, Inc. (St. Louis, MO USA) & Cat. \#: SAB4200100 \\
\hline Rabbit anti-FIP200 & Proteintech Group, Inc. (Rosemont, IL USA) & Cat. \#: 17250-1-AP \\
\hline Rabbit anti-AMPK $\beta$ & Novus Biologicals, Inc. (Littleton, CO USA) & Cat. \#: NBP1-87487 \\
\hline Rabbit anti-pLKB1 (Ser428) & Abcam, Inc. (Cambridge, MA USA) & Cat. \#: Ab63473 \\
\hline Rabbit anti-pAMPK (Thr172)-PE & BIOSS, Inc. (Woburn, MA USA). & Cat. \#: BS-4002RP \\
\hline Rabbit anti-pATG1 (Ser556)-PE & BIOSS, Inc. (Woburn, MA USA) & Cat. \#: ABIN746733 \\
\hline \multicolumn{3}{|l|}{ Fungal, Bacterial and Virus Strains } \\
\hline C. neoformans strain H99 & Dr. Xiaorong Lin (Texas A\&M University, College Station, TX) & $N / A$ \\
\hline C. neoformans strain Al100-dsRed & Dr. Xiaorong Lin (Texas A\&M University, College Station, TX) & $N / A$ \\
\hline C. neoformans strain cap59 & Dr. Xiaorong Lin (Texas A\&M University, College Station, TX) & N/A \\
\hline C. neoformans strain C177 & Dr. Jong, A. (University of California, Los Angeles, CA, USA) & $\mathrm{N} / \mathrm{A}$ \\
\hline C. neoformans strain $c p s 1 \Delta$ & Dr. Jong, A. (University of California, Los Angeles, CA, USA) & N/A \\
\hline C. neoformans strain C588 & Dr. Jong, A. (University of California, Los Angeles, CA, USA) & $\mathrm{N} / \mathrm{A}$ \\
\hline \multicolumn{3}{|c|}{ Chemicals, Peptides, and Recombinant Proteins } \\
\hline STO-609 & Sigma-Aldrich, Inc. (St. Louis, MO USA) & Cat. \#: S1318-5MG \\
\hline BAPTA-AM & Sigma-Aldrich, Inc. (St. Louis, MO USA) & Cat. \#: A1076-25MG \\
\hline KN62 & Sigma-Aldrich, Inc. (St. Louis, MO USA) & Cat. \#: I2142-1MG \\
\hline RIPA buffer & G-Biosciences (San Diego, CA USA) & Cat. \#: 786-489 \\
\hline Phosphatase inhibitor cocktail 2 & Sigma-Aldrich, Inc. (St. Louis, MO USA) & Cat. \#: P5726 \\
\hline Phosphatase inhibitor cocktail 3 & Sigma-Aldrich, Inc. (St. Louis, MO USA) & Cat. \#: P0044 \\
\hline Amino-polystyrene Particles & Spherotech, Inc. (Lake Forest, IL, USA) & Cat \#: AP-10-10 \\
\hline Hyaluronic acid & Santa Cruz Biotechnology, Inc. (Dallas, TX USA) & Cat \#: sc-337865 \\
\hline EDC hydrochloride & Santa Cruz Biotechnology, Inc. (Dallas, TX USA) & CAS \#: 25952-53-8 \\
\hline pENTR4-eGFP-C1 entry vector & Addgene (Cambridge, MA, USA) & Plasmid \#17396 \\
\hline
\end{tabular}




\begin{tabular}{|c|c|c|}
\hline pLenti-PGK-Neo destination vector & Addgene (Cambridge, MA, USA) & Plasmid \#19067 \\
\hline psPAX2 & Addgene (Cambridge, MA, USA) & Plasmids \#12260 \\
\hline pMD2G/VSV-G & Addgene (Cambridge, MA, USA) & Plasmids \#12259 \\
\hline G418 & Invivogen (San Diego, CA, USA) & Cat \#: NC9227938 \\
\hline Lipofectamine $^{\mathrm{TM}} 2000$ & Invitrogen (Carlsbad, CA, USA) & Cat \#: 11668027 \\
\hline \multicolumn{3}{|l|}{ Critical Commercial Assays } \\
\hline Phagocytosis Assay Kit (IgG FITC) & Cayman Chemical, Inc. (Ann Arbor, MI USA) & Cat. \#: 500290 \\
\hline Fe-NTA column & Thermo Fischer Scientific (Waltham, MA USA) & Cat. \#: 88300 \\
\hline Pierce ECL Plus chemiluminescence kit & Thermo Fischer Scientific (Waltham, MA USA) & Cat. \#: 32132X3 \\
\hline \multicolumn{3}{|l|}{ Experimental Models: Cell Lines } \\
\hline RAW264.7 cells & ATCC (Manassas, VA USA) & TIB-71 \\
\hline J774A.1 cells & ATCC (Manassas, VA USA) & TIB-67 \\
\hline GFP-Gal8 (RAW264.7 macrophages) & This work & NA \\
\hline GFP-LC3 (RAW macrophages) & $\begin{array}{l}\text { Dr. Douglas Green (St. Jude Children's Research Hospital, } \\
\text { Pittburgh, PA USA) }\end{array}$ & NA \\
\hline B6J2 cells & Dr. J. Suttles (University of Louisville, Louisville, KY USA) & (Sag et al., 2008) \\
\hline B6J2 cells (AMPK $\alpha-D N)$ & Dr. J. Suttles (University of Louisville, Louisville, KY USA) & (Sag et al., 2008) \\
\hline \multicolumn{3}{|c|}{ Experimental Models: Organisms/Strains } \\
\hline C57BL/6 wild-type (WT) mice & Jackson Laboratories, Inc. (Bar Harbor, ME USA) & Stock No: 000664 \\
\hline $\begin{array}{l}\text { CD44-KO mice (B6.129 (Cg)- } \\
\text { Cd44 }\end{array}$ & Jackson Laboratories, Inc. (Bar Harbor, ME USA) & Stock \#: 005085 \\
\hline
\end{tabular}

Further information and requests for resources and reagents should be directed to and will be fulfilled by the Lead Contact, Paul de Figueiredo (pjdefigueiredo@tamu.edu).

\section{EXPERIMENTAL MODEL DETAILS}

\section{Bone marrow-derived macrophage harvest and cultivation}

9 Bone marrow cells were collected from the femurs of littermate control and CD44 KO mice, and

10 cultivated in L929-cell conditioned media [DMEM medium containing 20\% L929 cell supernatant, 11 supplemented with $10 \%$ (v/v) FCS, penicillin $(100 \mathrm{U} / \mathrm{ml})$, and streptomycin $(100 \mathrm{U} / \mathrm{ml})]$. After 3 12 days of culture, non-adherent precursors were washed away and the retained cells were propagated

13 in fresh L929-cell conditioned media for another 4 days. For experimentation, BMDMs were split

14 in 24-well plates at a density of $2.5 \times 10^{5}$ cells per well in L929-cell conditioned media and 15 cultured at $37^{\circ} \mathrm{C}$ with $5 \% \mathrm{CO}_{2}$ overnight before use. 
Method Details

Cryptococcus Strains, Cell Culture, Cryptococcus Infection, and CFU Assay

4 Yeast forms of $\mathrm{Cn}$ cells were cultured on YPD (Difco $\left.{ }^{\mathrm{TM}}\right)$ agar plates and maintained on the plates

5 for 4 to 5 days prior to experimentation. Mammalian cell lines were routinely incubated in DMEM supplemented with $10 \% \mathrm{FBS}$ in a $5 \% \mathrm{CO}_{2}$ atmosphere at $37^{\circ} \mathrm{C}$. Preparation of cryptococcal and host cells for infection as well as CFU assays that measured internalized $\mathrm{Cn}$ cells were performed as previously described (Qin et al., 2011; Pandey et al., 2017).

\section{Immunofluorescence Microscopy Assays for Cn Phagocytosis}

11 Mammalian host cells were first cultivated on $12 \mathrm{~mm}$ glass coverslips (Fisherbrand) on the bottom 12 of 24-well plates (Falcon) for 12-16 h before infection. Next, the host cells were infected with the 13 tested $\mathrm{Cn}$ cells and incubated at $37^{\circ} \mathrm{C}$ in a $5 \% \mathrm{CO}_{2}$ atmosphere. At the indicated time points post14 infection, culture media was removed and the infected host cells were washed 6 to 8 times with 15 PBS ( $\mathrm{pH}$ 7.4) before being fixed with 3.7\% formaldehyde. The fixed cells were then processed Cn 16 phagocytosis assays using antibody (18B7) directed against $\mathrm{Cn}$ as previous described (Qin et al., 17 2011; Pandey et al., 2017) without adding Triton X-100 to the staining buffer. The numbers of 18 internalized (unstained) and extracellular (stained) Cn cells were then quantified and plotted. 19 Images represent a representative image from triplicate replicates, with 100 fields imaged per 20 replicate.

\section{Drug/compound Treatments}

23 Murine macrophage J774.A1 or RAW264.7 cells were overnight cultured in 48 well plates and 24 then coincubated with assorted pharmacological compounds, including KN62, STO-609 or 25 BAPTA-AM for $3 \mathrm{~h}$. The media containing the compounds was then removed. For CFU assays, 26 the drug-treated cells were extensively washed with fresh medium and then infected with $\mathrm{Cn}$ cells.

27 At 3 h.p.i., the infected cells were lysed and performed CFU assay as previously described (Qin 28 et al., 2011; Pandey et al., 2017). For western blots, the compound-treated cells were washed 3 29 times with cold $1 \times \mathrm{PBS}, \mathrm{pH} 7.4$, lysed and performed immunoblotting assay as previously 30 describe (Pandey et al., 2017). 


\section{Lentivirus-Mediated Depletion of Host Proteins}

2 The pSuperRetro retrovirial vector system (OligoEngine, Inc.) was used to knockdown target gene expression in murine cells according to the manufacturer's instructions. The oligonucleotides used for shRNA construction to knockdown the expression of mouse genes and the accompanying references are listed in Table S1. Transfection was performed in 6-well plates containing $1.5 \times 10^{5}$ RAW264.7 or B6J2 cells. Clones with the insert stably integrated were selected with puromycin. Western blot was performed to validate the depletion of the targeted proteins. All

8 Westerns were performed in triplicate and representative findings are shown.

\section{Generation of GFP-tagged Gal8 RAW264.7 cells}

11 The GFP-galectin-8 expression construct was made by first cloning the cDNA sequence of Lgals8 12 (from RAW 264.7 cells) into the pENTR4-eGFP-C1 entry vector (Campeau et al., 2009), resulting 13 in a fusion of eGFP on the N-terminus of galectin-8. This construct was fully Sanger sequenced 14 (Eton Biosceinces, San Diego, CA) to verify the fusion protein was in-frame and error-free. GFP15 Gal8 was then Gateway cloned with LR Clonase (Invitrogen) into the pLenti-PGK-Neo 16 destination vector (Campeau et al., 2009). Lenti-X 293T cells (Takara Bio) were co-transfected 17 with pLenti-GFP-Gal8 and the packaging plasmids psPAX2 and pMD2G/VSV-G (Addgene 18 Plasmids \#12259-60) to produce lentiviral particles. RAW 264.7 cells were transduced with GFP19 Gal8 lentivirus for two consecutive days plus 1:1000 Lipofectamine 2000 (Invitrogen) and 20 selected for 5 days with $750 \mu \mathrm{g} / \mathrm{ml} \mathrm{G} 418$ (Invivogen). Expression of GFP-Gal8 was confirmed by

21 Western blot analysis and fluorescence microscopy.

\section{Confocal Microscopy Assays}

24 Host cells were infected with live or heat killed, opsonized or unopsonized capsular or acapsular 25 strains of $\mathrm{Cn}$. At the indicated times post-infection, host cells were fixed for confocal 26 immunofluorescence microscopy analysis using antibodies directed against the indicated host 27 proteins. Immunofluorescence microscopy staining and imaging methods (Qin et al., 2008; Qin 28 et al., 2011; Pandey 2017; Pandey 2018 ) were used to determine the subcellular localization of 29 host AIC components in infected host cells. Samples were observed on a laser scanning confocal 30 microscope or on a confocal fluorescence microscope (ECLIPSE Ti, Nikon). Confocal images $31(1,024 \times 1,024$ pixels $)$ were acquired and processed with NIS elements AR 3.0 software (Nikon) 
1 and assembled with Adobe Photoshop CC 2019 (Adobe Systems, CA, USA). Digital image 2 analysis and quantification was performed as previously described (Qin et al., 2011). Findings 3 from our subcellular localization analyses were not an artifact of secondary antibody cross 4 reactivity with host or pathogen components because negligible fluorescence signal was observed 5 when infected cells were stained with secondary antibodies alone, or the pathogen alone was stained with the antibodies used in the experiments (Figure S4G).

\section{Protein pull-down assay with HA-coated or naked beads}

9 Spherical amino polysterene beads (size: 1.0-1.4 $\mu \mathrm{m}$, Spherotech Inc, IL, USA) were covalently 10 coupled using EDC [N-Ethyl- $N^{\prime}$-(3-dimethylaminopropyl)carbodiimide hydrochloride] to 11 hyaluronic acid (HA) (Santa Cruz Biotec.) as per the manufacturer's (Spherotech) instruction. 12 Briefly, $200 \mu \mathrm{l}$ of $0.05 \mathrm{M}$ sodium acetate buffer ( $\mathrm{pH} 5.0$ ), $2 \mathrm{mg}$ of HA, $2 \mathrm{ml}$ of 5\% w/v Amino 13 particles and $20 \mathrm{mg}$ of EDC were mixed in a glass centrifuge tube. The contents were vortexed 14 and incubated for $2 \mathrm{hrs}$ at ambient temperature on a rotary mixer. Following incubation, the tube 15 was centrifuged at $3000 \times \mathrm{g}$ for 15 minutes and supernatant was carefully discarded. The pellet was washed twice in $1 \times \mathrm{PBS}$ and resuspended in $2 \mathrm{ml}$ of $1 \times \mathrm{PBS}$ to obtain $5 \% \mathrm{w} / \mathrm{v}$ suspension of

17 HA coated beads. RAW 264.7 cells $\left(3 \times 10^{5}\right)$ were washed with $1 \times$ PBS. Next, a bead incubation 18 solution containing $5 \mu \mathrm{l}$ of HA-coated or naked beads in $200 \mu \mathrm{l}$ of PBS was added to the washed 19 cells. The cells were centrifuged for $10 \mathrm{~min}$ at 1500 RPM and then incubated in a humidified 20 incubator containing $5 \% \mathrm{CO}_{2}$ for $2 \mathrm{hrs}$. The treated cells were washed 2-4 times with ice-cold 1

$21 \times$ PBS and lysed in $80 \mu \mathrm{l}$ RIPA buffer supplemented with a cocktail of protease and phosphatase 22 inhibitors. Beads were separated from the lysate by high-speed centrifugation at $4^{\circ} \mathrm{C}$. The 23 separated beads were washed 3 times with RIPA buffer and finally resuspended in $80 \mu$ RIPA 24 buffer and $20 \mu \mathrm{l}$ of $5 \times$ sample buffer (Thermo Scientific) and boiled for 5 mins. Samples were 25 finally resolved by SDS-PAGE and subjected to western blot analysis.

\section{Immunoblotting Analysis}

28 Preparation of protein samples and western blot analysis were performed as described previously 29 (Qin et al., 2011; Pandey et al., 2017; Pandey et al., 2018). Blot densitometry was performed using 30 the ImageJ (http://rsbweb.nih.gov/ij/) software package. All Westerns were performed in triplicate 31 and representative findings are shown. 


\section{Quantification and Statistical Analysis}

3 The quantitative data presented in this work represent the mean \pm standard deviation (SD) from at 4 least three independent experiments. To easily compare results from independent experiments, the 5 data from controls, such as protein expression level, blot densitometry, CFU, intracellular Cn

6 number, etc., were normalized as 1 or $100 \%$. The significance of the data was assessed using the

7 Student's $t$-test to assess statistical significance between two experimental groups or a one-way

8 ANOVA test to evaluate the statistical differences of multiple comparisons of the data sets. 


\section{Supplemental Figures and Figure Legends}

\section{Interactions between fungal hyaluronic acid and host CD44 promote internalization by recruiting host autophagy proteins to forming phagosomes}

Sheng Li Ding ${ }^{1,2,6}$, Aseem Pandey ${ }^{2,3,6}$, Xuehuan Feng ${ }^{2,6}$, Jing Yang ${ }^{2}$, Luciana Fachini da Costa ${ }^{2,4}$, Roula Mouneimne ${ }^{3}$, Allison Rice-Ficht ${ }^{4}$, Samantha L.Bell ${ }^{5}$, Robert O. Watson ${ }^{5}$, Kristin Patrick ${ }^{5}$, Qing-Ming Qin ${ }^{1,2^{*}}$, Thomas A. Ficht ${ }^{3 *}$, Paul de Figueiredo ${ }^{2,3,7^{*}}$

${ }^{1}$ College of Plant Sciences \& Key Laboratory of Zoonosis Research, Ministry of Education, Jilin University, Changchun 130062, Jilin, China; Department of Plant Pathology, College of Plant Protection, Henan Agricultural University, Zhengzhou 450002, Henan, China

${ }^{2}$ Department of Microbial Pathogenesis and Immunology, Texas A\&M Health Science Center, Norman Borlaug Center, Texas A\&M University, College Station, Texas 77843, USA

${ }^{3}$ Department of Veterinary Pathobiology, Texas A\&M University, College Station, Texas 77843, USA

${ }^{4}$ Department of Molecular and Cellular Medicine, College of Medicine, Texas A\&M Health Science Center, College Station, Texas 77843, USA

${ }^{5}$ Department of Microbial Pathogenesis and Immunology, Texas A\&M Health Science Center, Bryan, Texas 77807, USA

${ }^{6}$ These authors contributed equally

${ }^{7}$ Lead contact

* Correspondence: qmqin@jlu.edu.cn (Q.M.Q.), tficht@tamu.edu (T.A.F.), pjdefigueiredo@tamu.edu (P.d.F.) 

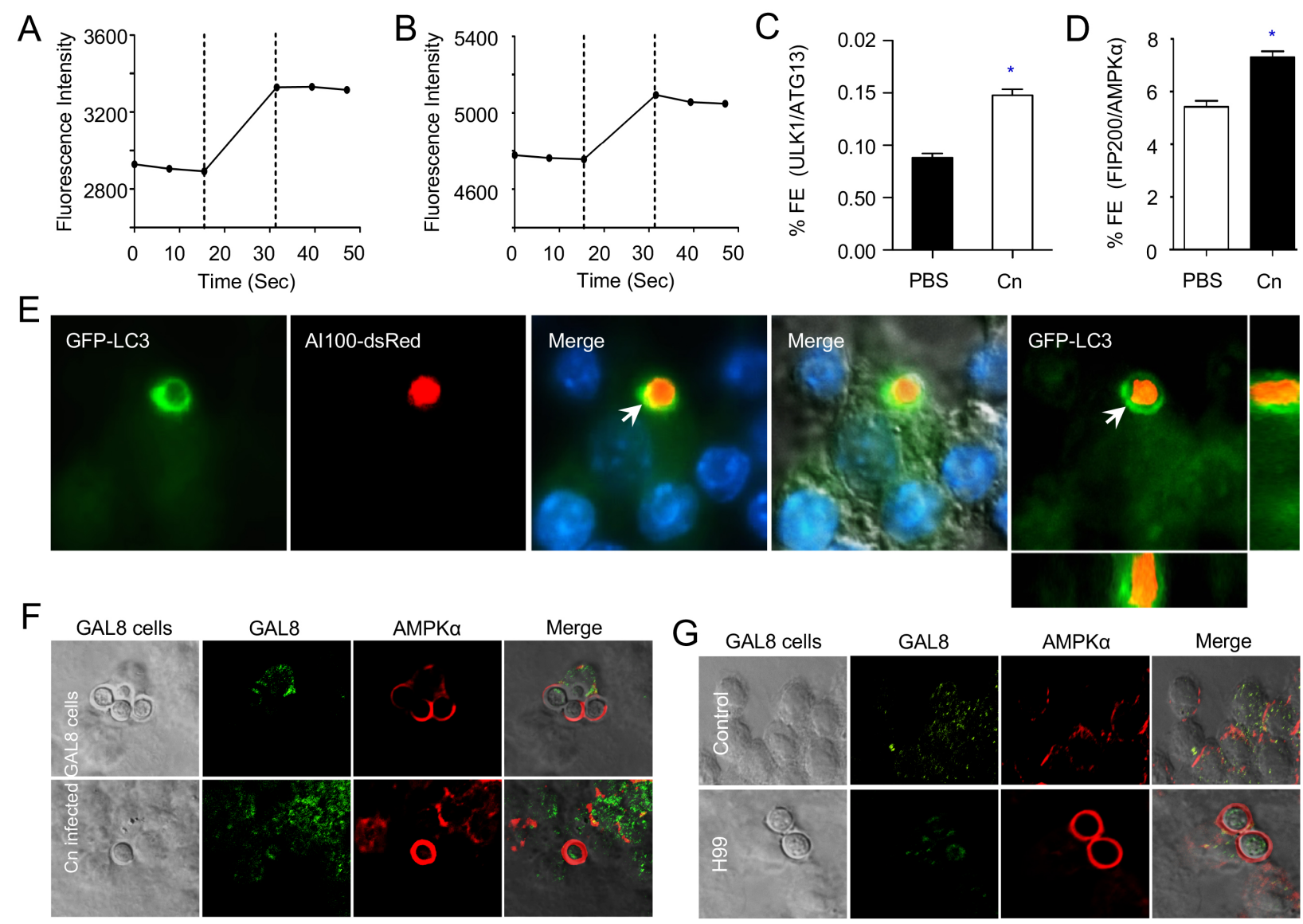

AMPKa
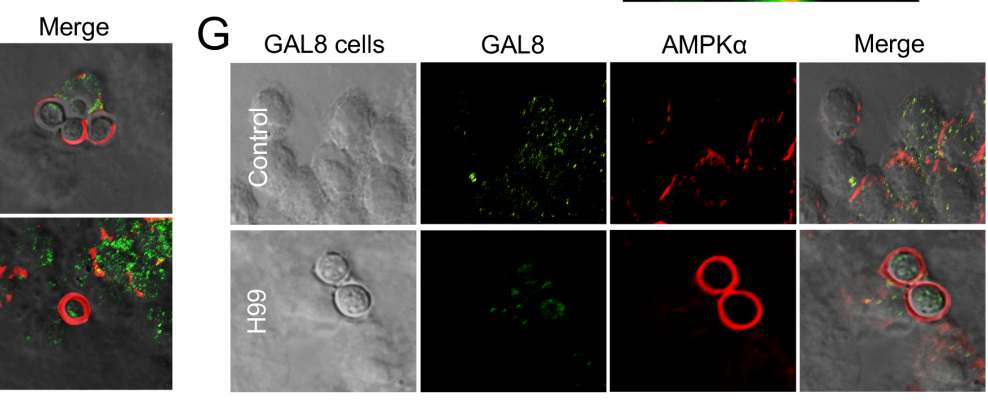

$\mathrm{H}$

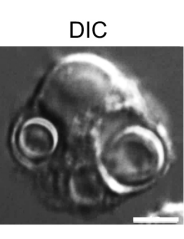

ULK1
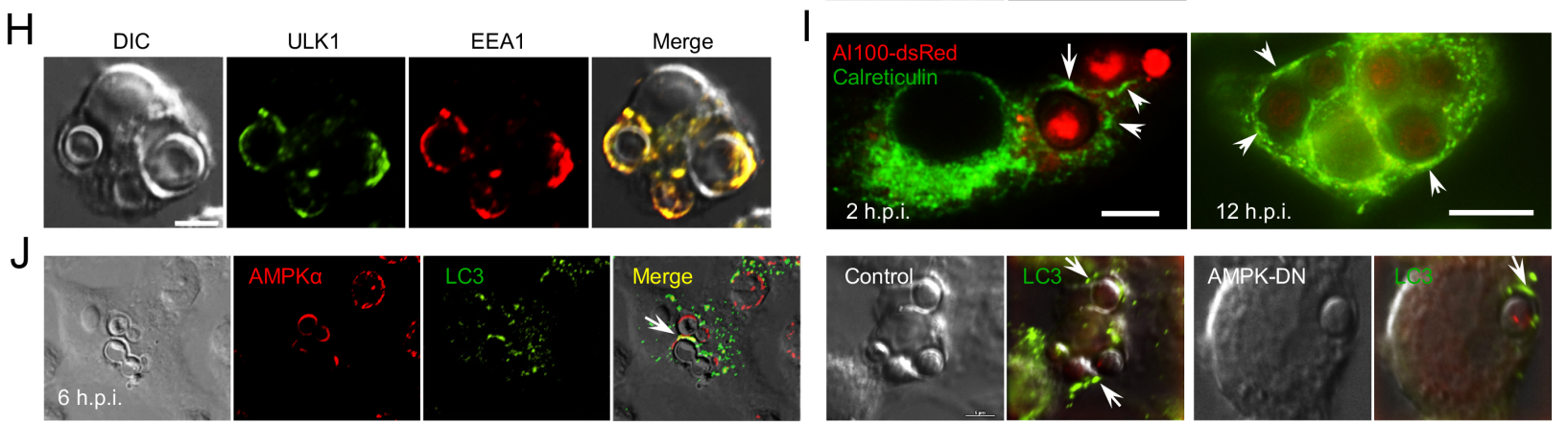

K

Wild-type B6J2 cells
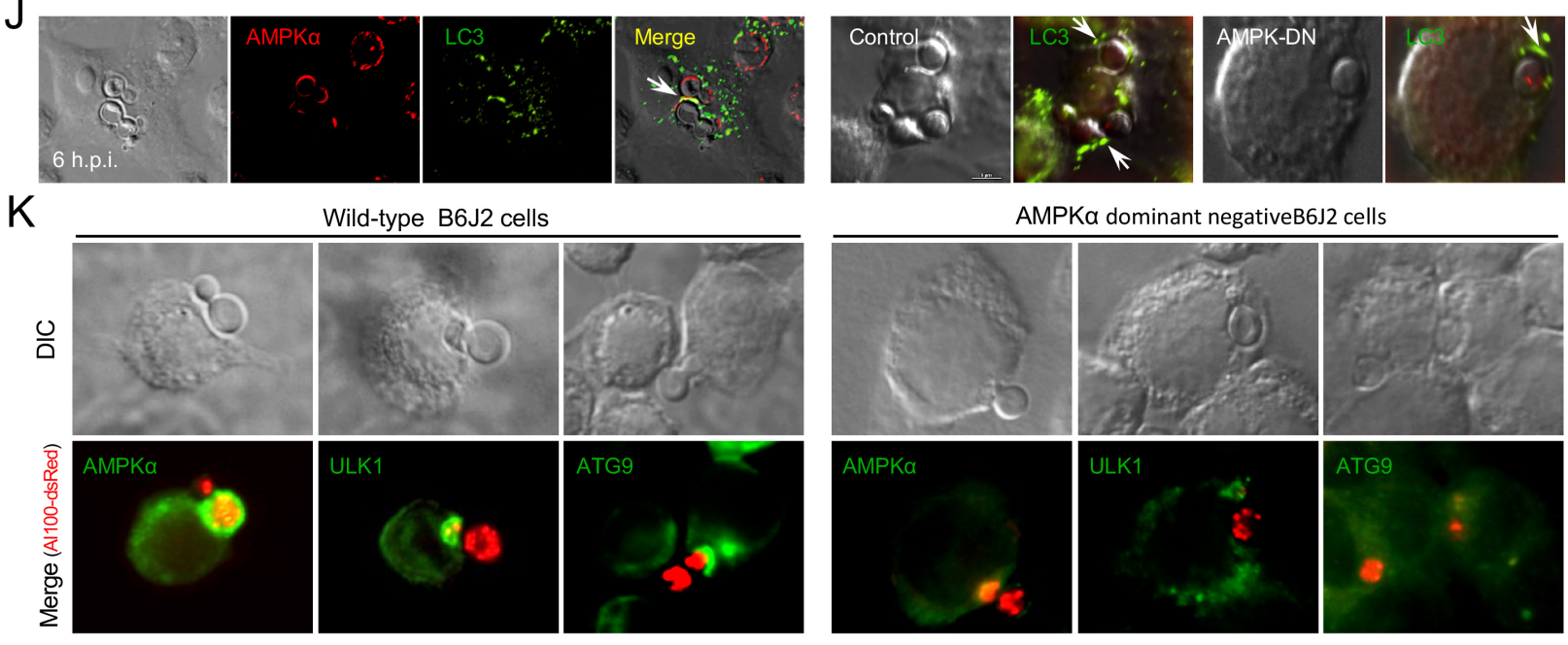
Figure S1. Recruitment of AIC components, but not galection 8, to nascent phagosomes during $\mathrm{Cn}$ internalization.

(A, B) Fluorescence intensity of ULK1 and ATG13 (A) or FIP200 and AMPK $\alpha$ (B) during Cn internalization.

(C, D) Interactions between host ULK1 and ATG13 (C) or FIP200 and AMPK $\alpha$ (D) recruited to Cn-containing vacuoles (CnCVs) in RAW264.7 macrophages. The infected host cells were quantified using FRET analysis. Data represent the means \pm standard deviations (SD) from at least three independent experiments. * indicates significance at $\mathrm{P}<0.05$.

(E) Recruitment of host LC3 to the nascent CnCVs during Cn internalization.

$(\mathrm{F}, \mathrm{G})$ Host galectin 8 is not recruited to nascent phagosomes containing $\mathrm{Cn}$ cells during $\mathrm{Cn}$ internalization by host cells expressing GFP-galectin 8 (F) or cells supplemented with UBEI-41, a cell permeable inhibitor of ubiquitin-activating enzyme E1 $(\mathrm{G})$.

(H) Colocalization of host early endosomal marker EEA1 with AIC component ULK1 surrounding nascent CnCVs.

(I) Recruitment of host endoplasmic reticulum marker calreticulin to nascent and formed CnCVs.

(J) Recruitment of host AMPK $\alpha$ and LC3 to nascent phagosomes containing Cn cells in infected bone marrow derived macrophages (BMDMs, left panel) or in B6J2 macrophages expressing dominant negative variant of AMPK $\alpha$ (AMPK-DN) and control (right panel).

(K) Less recruitments of host AMPK $\alpha$ and AIC component ULK1 or ATG9 to nascent CnCVs in B6J2 macrophages expressing dominant negative AMPK $\alpha$ variant during $\mathrm{Cn}$ internalization. 
A

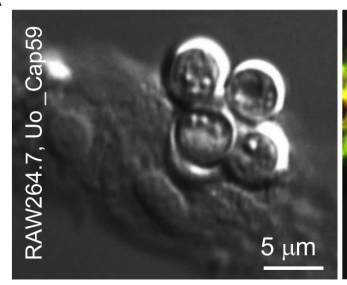

C

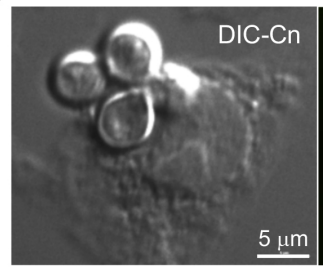

D

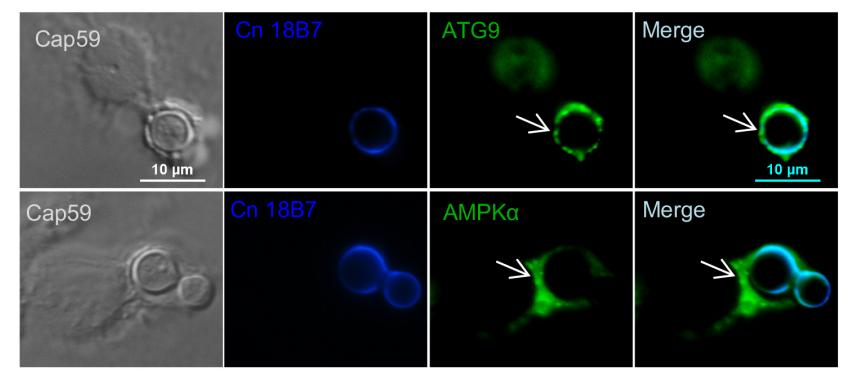

B
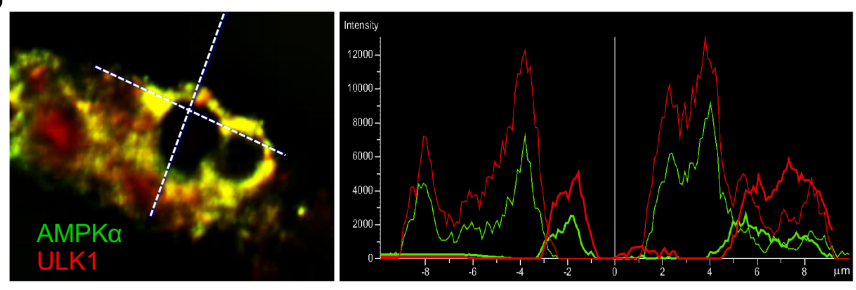

E

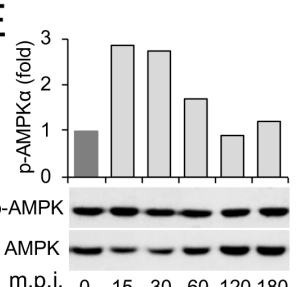

F

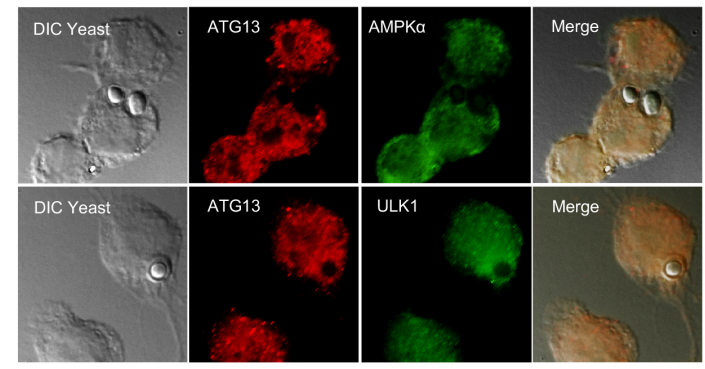

G

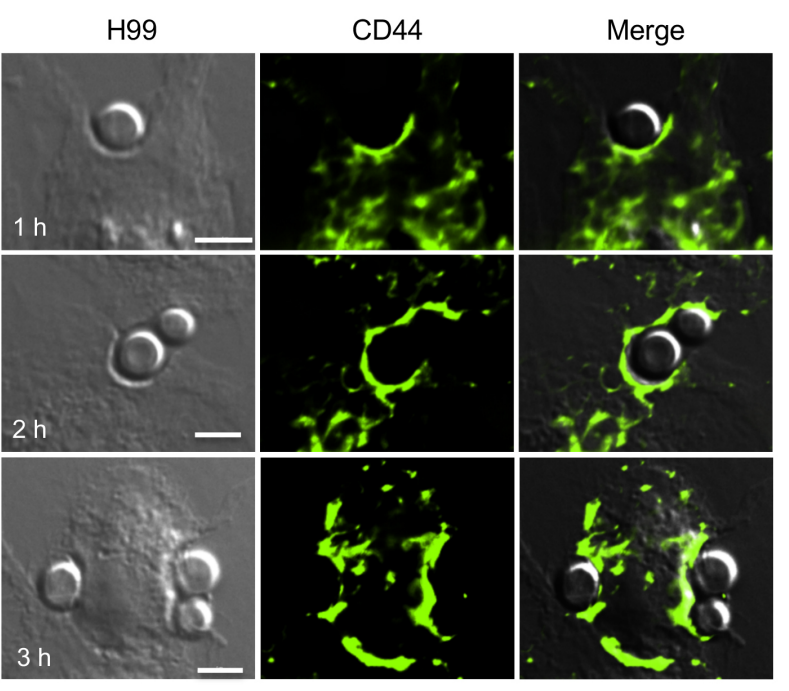

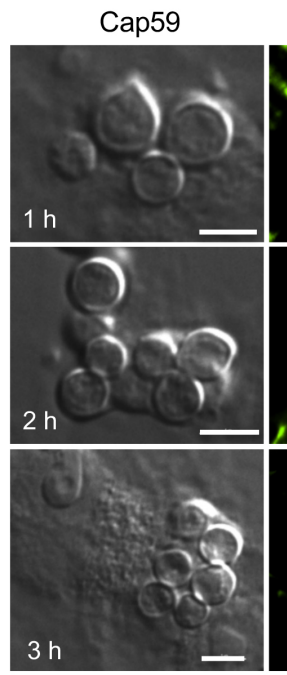

CD44

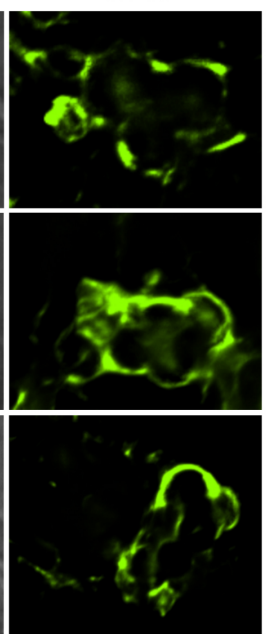


Figure S2. Recruitment of host AIC components and CD44 to forming or nascent CnCVs during Cn internalization.

(A) Recruitment of AMPK $\alpha$ and AIC components ULK1 to nascent CnCVs.

(B) The fluorescence intensity profile of AMPK $\alpha$ (green) and ULK1 (red) along the two crossed white lines (left panel).

(C) Colocalization of ATG9 with ATG13 in the vicinities of CnCvs.

(D) Colocalization of host AMPK $\alpha$ or ATG9 with cryptococcal glucuronoxylomannan (GXM)-specific monoclonal antibody $18 \mathrm{~B} 7$.

(E) Activation of hos AMPK $\alpha$ by heat-killed (HK) Cn cells (cap 59) during phagocytosis. Representative results from one of three independent experiments are shown.

(F) Recruitment of AIC components and AMPK $\alpha$ to nascent phagosomes is hardly detected during phagocytosis of yeast (Saccharomyces cerevisiae) cells by host cells.

(G) Recruitment of CD44 during a time course (3 hr) of Cn internalization. 

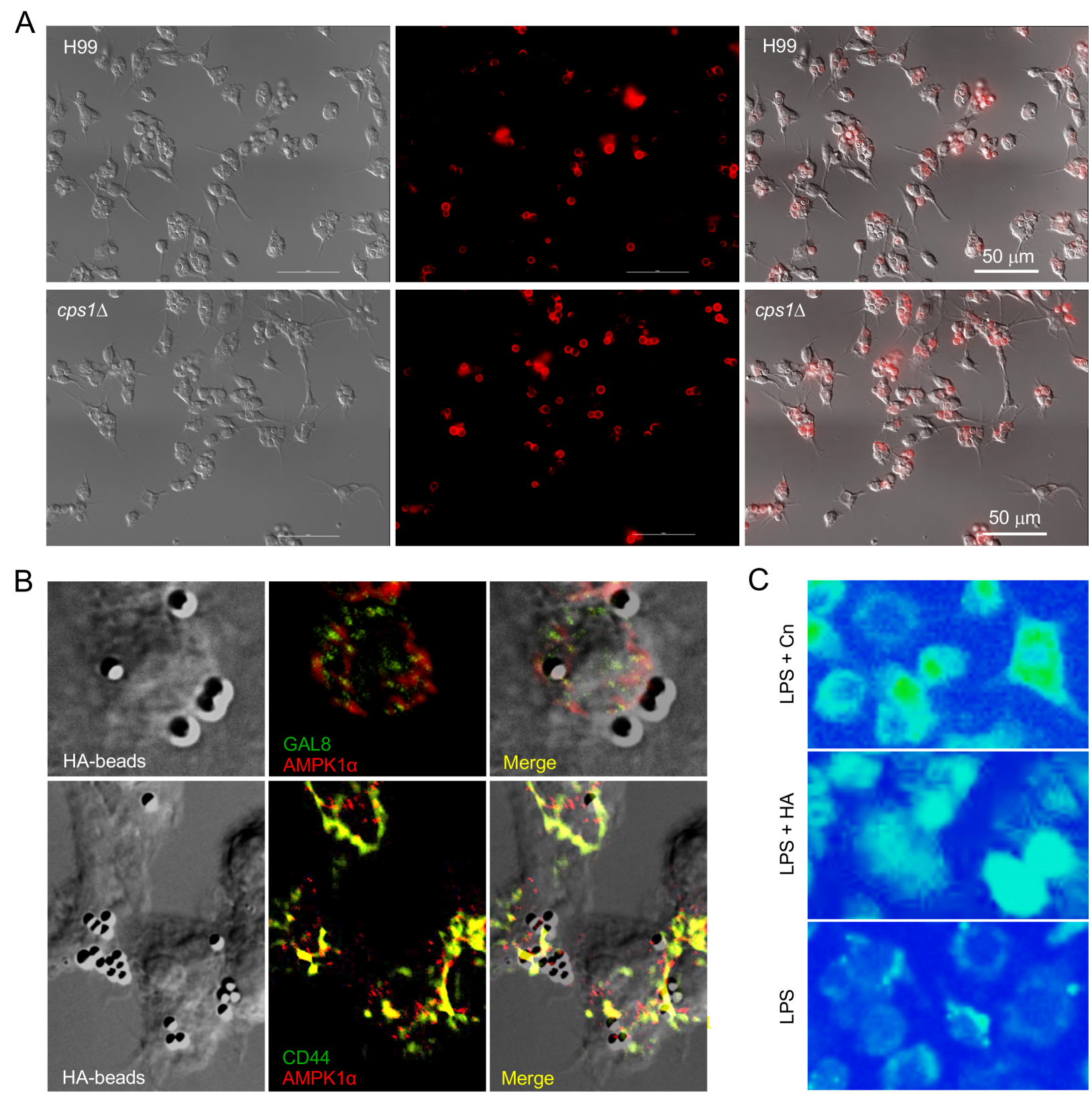

Figure S3. Fungal hyaluronic acid (HA) is required for $\mathrm{Cn}$ internalization.

(A) Disruption of Cn cpsl impairs internalization of the fungal pathogen (related to Figure 3B-C).

(B) Recruitment of CD44, not galectin 8, to the forming or nascent phagosomes associated with HA-coated beads. Upper panel: localization of galectin 8 and AMPK $\alpha$ in cells incubated with HAcoated beads. Low panel: colocalization of CD44 and AMPK $\alpha$ to the forming or nascent phagosomes related to HA-coated beads.

(C) Incubation of host cell with Cn harboring cps1 (top) or HA (middle) increases intracellular $\mathrm{Ca}^{2+}$ level (exciated at $340 \mathrm{~nm}$ ). 

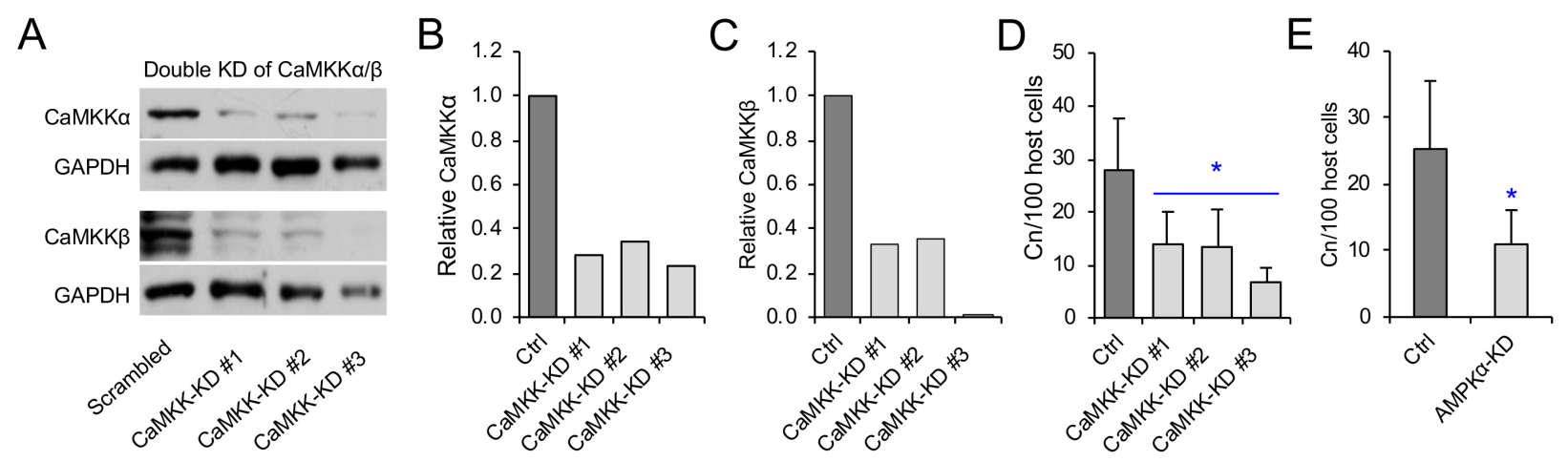

$\mathrm{F}$

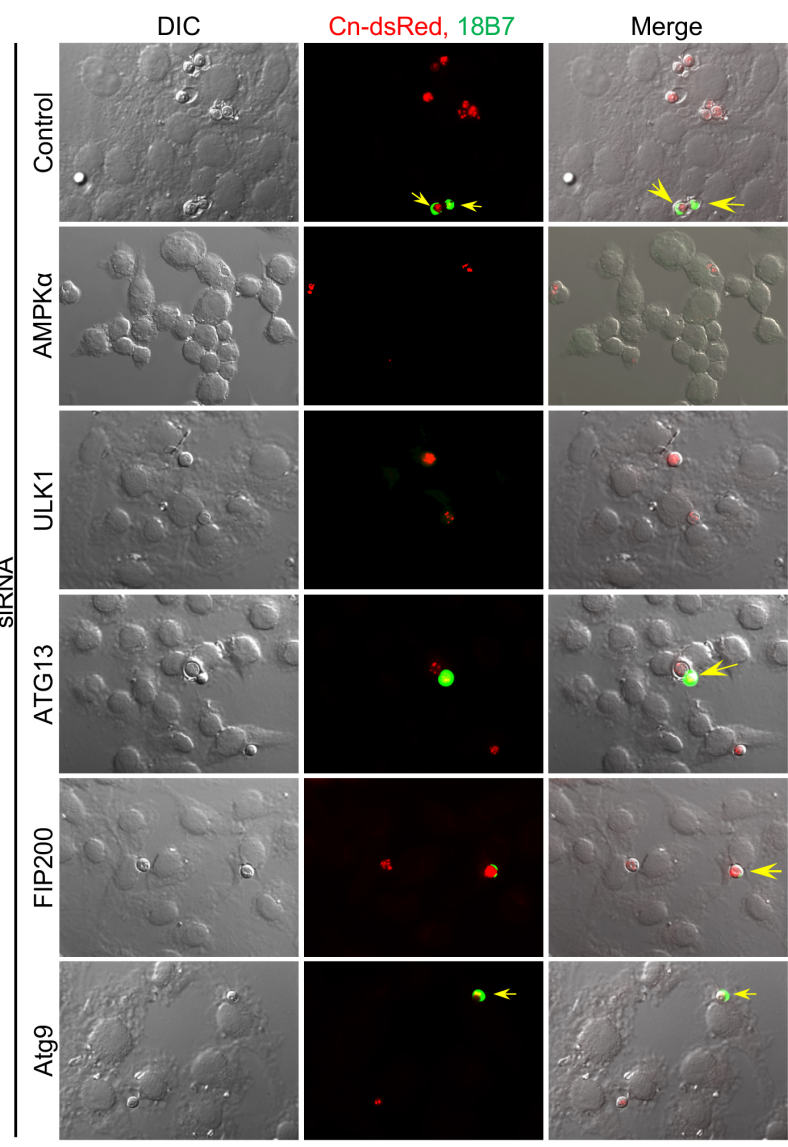

G
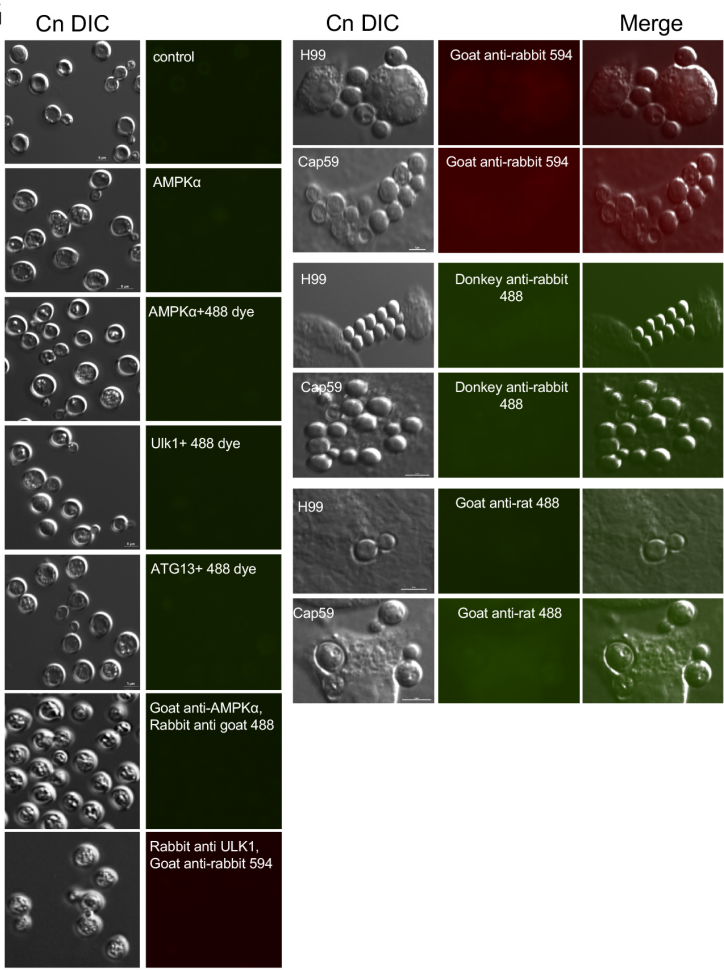
Figure S4. Components in the CaMKK -AMPK-ULK1 signal aix play important roles in Cn internalization.

(A) Immunobloting assay to test the depletion of CaMKK $\alpha / \beta$ protein level by the shRNA approach. $(\mathrm{B}, \mathrm{C})$ Relative protein expression levels of CaMKK $\alpha(\mathrm{B})$ or $\mathrm{CaMKK} \beta(\mathrm{C})$ in RAW macrophages transfected with scambled or CaMKK $\alpha / \beta$ shRNAs . Data from a representative experiment shown in (A).

(D, E) Depletion of host cell CaMKK $\alpha / \beta$ (D) or AMPK $\alpha$ (E) reduces $\mathrm{Cn}$ internalization determined by immunofluorescence microscopy assay. Data represent the means \pm SD from three independent experiments. *: significance at $\mathrm{p}<0.05$.

(F) Depletion of host cell AMPK $\alpha$ and AIC components reduces Cn internalization determined by immunofluorescence microscopy assay. Images from a representative experiment of three independent experiments.

(G) Cn cells harvested from infected host cells (leaf panel) or during host infection (right panel) do no display cross activities with the primary or secondary antibodies used in this work. 
Table S1. shRNAs and PCR primers used in this study

\begin{tabular}{|c|c|c|}
\hline shRNA ID & Sequence (5'-3') & References \\
\hline shCaMKKa 1 & GGAAGTGCCCGTTCATTGATT & This study \\
\hline shCaMKKa 2 & TCAATGGCTGAGGTGAGGCAC & This study \\
\hline shCaMKK $\beta 1$ & GGTGCTGTCCAAAAAGAAA & This study \\
\hline shCaMKK $\beta 2$ & TTGCGTAATAAGTATTGTCAT & This study \\
\hline \multirow{2}{*}{ shFIP200 } & GAGAGAACTTGTTGAGAAA & Pandey et al., 2017 \\
\hline & ACATGAAGGCTCAGAGAAA & ditto \\
\hline \multirow{2}{*}{ shUlk1 } & GAGCAAGAGCACACGGAA A & ditto \\
\hline & AGACTCCTGTGACACAGAT & ditto \\
\hline \multirow{2}{*}{ shAtg13 } & GAGAAGAATGTCCGAGAAT & ditto \\
\hline & ACAGGAAGGACTTGGACAA & ditto \\
\hline \multirow{2}{*}{ shPrkaa1 } & TGAATTAAACCCACAGAAA & ditto \\
\hline & GGTCATCAGTACACCATCT & ditto \\
\hline \multirow{2}{*}{ shPrkab1 } & TGAACAAGGACACGGGCAT & ditto \\
\hline & GCACGACCTGGAAGCGAAT & ditto \\
\hline Scramble 1 & ATTGTATGCGATCGCAGAC & ditto \\
\hline Scramble 2 & CACCAGCATCTGATCTAGA & ditto \\
\hline Ulk1-F1 & ACTGCGGCCGCatggattacaaggatgacgatgacaagatggag ccgggccgcggcggc & ditto \\
\hline Ulk1-r1 & GAAGAATTCtcaggcatagacaccactcag & ditto \\
\hline ULK1-F2 & GATCTGGATCCGGAGTCGACGGAGCGGCCGCatggatta caagga & ditto \\
\hline Ulk1-R2 & AGCGCCTCCCCTACCCGGTAGAATTCtcaggcatagaca ccactc & ditto \\
\hline Atg13-F1 & $\begin{array}{l}\text { ACTAGATCTATGTACCCATACGATGTTCCAGATTACGCT } \\
\text { ATGGAAACTGAACTCAGCTCC }\end{array}$ & ditto \\
\hline Atg13-R1 & GAACTCGAGTTACTGCAGGGTTTCCACAAA & ditto \\
\hline Atg13-F2 & ACTCCTTCTCTAGGCGCCGGAATTAGATCTATGTACCCA TACGAT & ditto \\
\hline Atg13-R2 & ACCCGGTAGAATTCGTTAACCTCGAGttactgcagggtt tccaca & ditto \\
\hline Prkaa1-F1 & ACTGCGGCCGCATGGTGAGCAAGGGCGAGGAGCTGTTCA & ditto \\
\hline Prkaa1-R1 & GAAGAATTCTTACTGTGCAAGAATTTTAAT & ditto \\
\hline Prkaa1-F2 & GATCTGGATCCGGAGTCGACGGAGCGGCCGCATGGTGAG CAAGGG & ditto \\
\hline Prkaa1-R2 & AGCGCCTCCCCTACCCGGTAGAATTCttactgtgcaaga atttta & ditto \\
\hline
\end{tabular}

